\title{
ORIGINAL
}

\author{
B. Woodward • M. Kashtalyan
}

\section{Three-dimensional elasticity analysis of sandwich panels with functionally graded transversely isotropic core}

Received: 4 March 2019 / Accepted: 12 August 2019 / Published online: 20 September 2019

(C) The Author(s) 2019

\begin{abstract}
In this paper, three-dimensional elastic deformation of rectangular sandwich panels with functionally graded transversely isotropic core subjected to transverse loading is investigated. An exponential variation of Young's and shear moduli through the thickness is assumed. The approach uses displacement potential functions for transversely isotropic graded media and a three-dimensional elasticity solution for a transversely isotropic graded plate developed by the authors. The effects of transverse shear modulus, loading localisation, panel thickness and anisotropy on the stresses and displacements in the panel are examined and discussed.
\end{abstract}

Keywords Three-dimensional elasticity $\cdot$ Sandwich panel $\cdot$ Functionally graded material $\cdot$ Analytical modelling

\section{Introduction}

Sandwich panels comprising two thin face sheets of high strength and stiffness, separated by a core of lower density and strength, are ideally suited to a variety of industrial applications, where high specific stiffness and strength are required, including aerospace, energy, transportation, marine and civil engineering. Modern trends in theoretical developments, novel designs and modern applications of sandwich structures are outlined in the recent reviews by Birman and Kardomateas [9] and Vescovini et al. [28].

Due to the mismatch in stiffness properties between the face sheets and the core, the interface between them is often the most vulnerable part of the sandwich panel. Sandwich panels are susceptible to delamination, caused by high interfacial stresses, especially under localised loading [1]. One effective method of minimising the large interfacial shear stresses is to make use of the functionally graded material concepts for the panel core [7]. Sandwich panels with graded core have been studied analytically, numerically and experimentally by many researchers, including Anderson [5], Kirugulige et al. [20], Zhu and Sankar [38], Apetre et al. [6], Kashtalyan and Menshykova [17], Etemadi et al. [14], Rahmani et al. [23], Wang et al. [29], Woodward and Kashtalyan [30,31,33,34], Sburlati [24], Zhu et al. [37], Alibeigloo [3], Alibeigloo and Liew [4], Liu et al. [21] and Xu et al. [36] . A detailed review of these studies can be found in Woodward and Kashtalyan [34].

More recently, Sburlati et al. [25] investigated the effect of functionally graded interlayers on bending response of circular sandwich panels in the context of elasticity theory using displacement potentials method. Kelly et al. [19] studied sandwich panels with graded core experimentally and found that grading the density of the foam cores mitigates through-thickness crack propagation and damage in the higher density foam layers. Xiao et al. [35] investigated the effect of stiffness grading on energy dissipation under indentation of sandwich panels with graded metallic cellular core. Bending, free vibration and buckling of sandwich

B. Woodward · M. Kashtalyan $(\varangle)$

Centre for Micro- and Nanomechanics (CEMINACS), School of Engineering, University of Aberdeen, Fraser Noble Building, Aberdeen AB24 3UE, UK

E-mail: m.kashtalyan@abdn.ac.uk 
panels with graded core resting on elastic foundation were examined by Akavci [2] using a new hyberbolic shear and normal deformation theory. Daynes et al. [12] proposed functionally graded core designs based on lattice beam diameter tailoring and lattice cell spatial tailoring. Both stiffness and strength of the optimised cores significantly increased compared to the uniform benchmark core. Birman and Costa [8] and Birman and Vo [10] investigated wrinkling of sandwich panels with graded core demonstrating that use of graded core increases wrinkling stability of sandwich panels. An extended higher-order approach to analysis of wrinkling in sandwich panels with graded core was proposed in Frostig et al. [15]. A new analytical method for solving exact three-dimensional equilibrium equations for functionally graded structures, including sandwich panels with graded core, was proposed by Brischetto [11].

The majority of analytical studies on sandwich panels with graded core found in the literature assume the core material to be isotropic. With many core materials being orthotropic or transversely isotropic in nature, the combined effect of transverse isotropy and stiffness gradient has not been fully explored yet. Three-dimensional analytical modelling of transversely isotropic materials represents a formidable challenge even in the absence of a stiffness gradient due to the complexity of the governing equations involved [26,27].

In this paper, the three-dimensional elasticity solution for transversely isotropic plate with exponential variation of the Young's modulus through the thickness [32] is extended to sandwich panels. The paper is organised as follows. In Sects. 2 and 3, problem statement and method of solution are presented, respectively. In Sect. 4, validation of the model through both comparison with results from the literature and a finite element study is presented. In Sect. 5, effects of anisotropy and stiffness gradient on stresses and displacements in the panel are presented and discussed.

\section{Problem statement}

A sandwich panel (Fig. 1) of length $a$, width $b$ and total thickness $h_{0}=2 h$ is referred to as a Cartesian co-ordinate system $x_{1}, x_{2}, x_{3}\left(0 \leq x_{1} \leq a, \quad 0 \leq x_{2} \leq b, \quad-h \leq x_{3} \leq h\right)$ and assumed to be symmetric with respect to the mid-plane $x_{3}=0$, with the face sheet thickness $h_{\mathrm{f}}$ and the core thickness $2 h_{\mathrm{c}}$. The core is subdivided into two layers for the sake of convenience.

The face sheets (layers 1 and 4 ) and the core (layers 2 and 3 ) are assumed to be inhomogeneous transversely isotropic materials with the $x_{3}$-axis as axis of material symmetry. Constitutive equations for each layer $(k=$ $1, \ldots, 4)$ of the panel are:

$$
\begin{aligned}
& \sigma_{11}^{(k)}=c_{11}^{(k)} \varepsilon_{11}^{(k)}+c_{12}^{(k)} \varepsilon_{22}^{(k)}+c_{13}^{(k)} \varepsilon_{33}^{(k)} \\
& \sigma_{22}^{(k)}=c_{12}^{(k)} \varepsilon_{11}^{(k)}+c_{11}^{(k)} \varepsilon_{22}^{(k)}+c_{13}^{(k)} \varepsilon_{33}^{(k)} \\
& \sigma_{33}^{(k)}=c_{13}^{(k)} \varepsilon_{11}^{(k)}+c_{13}^{(k)} \varepsilon_{22}^{(k)}+c_{33}^{(k)} \varepsilon_{33}^{(k)} \\
& \sigma_{23}^{(k)}=2 c_{44}^{(k)} \varepsilon_{23}^{(k)} \\
& \sigma_{13}^{(k)}=2 c_{44}^{(k)} \varepsilon_{13}^{(k)} \\
& \sigma_{12}^{(k)}=2 c_{66}^{(k)} \varepsilon_{12}^{(k)}=\left(c_{11}^{(k)}-c_{12}^{(k)}\right) \varepsilon_{12}^{(k)}
\end{aligned}
$$

where $\sigma_{i j}^{(k)}, \varepsilon_{i j}^{(k)}$ are components of the stress and strain tensors and $c_{11}^{(k)}, c_{12}^{(k)}, c_{13}^{(k)}, c_{33}^{(k)}, c_{44}^{(k)}$ are five independent elastic coefficients, which in general case depend on $x_{1}, x_{2}, x_{3}$. It is assumed that in each layer

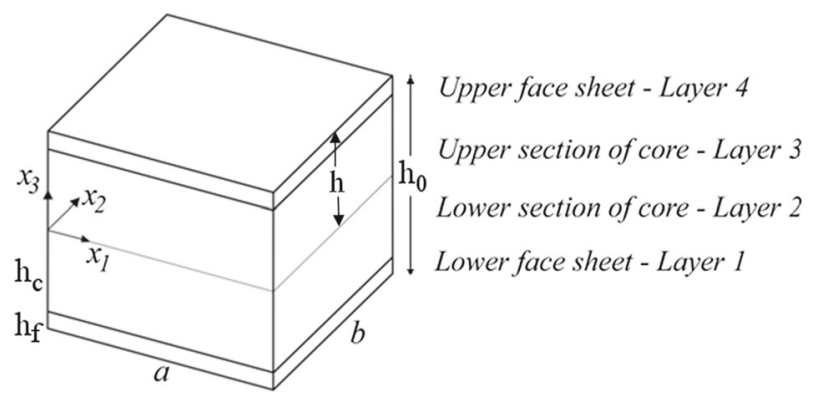

Fig. 1 Sandwich panel relative to Cartesian coordinates, with the core subdivided into two layers 
of the panel, elastic coefficients have the same functional dependence on the transverse co-ordinate $x_{3}$ :

$$
\begin{aligned}
c_{11}^{(k)} & =c_{11}^{0(k)} \tilde{m}\left(x_{3}\right) \\
c_{12}^{(k)} & =c_{12}^{0(k)} \tilde{m}\left(x_{3}\right) \\
c_{13}^{(k)} & =c_{13}^{0(k)} \tilde{m}\left(x_{3}\right) \\
c_{33}^{(k)} & =c_{33}^{0(k)} \tilde{m}\left(x_{3}\right) \\
c_{44}^{(k)} & =c_{44}^{0(k)} \tilde{m}\left(x_{3}\right)
\end{aligned}
$$

This is equivalent to assuming that within each layer, Young's and shear moduli depend on the transverse coordinate, whilst Poisson's ratios are constant [32].

It is also assumed that the elastic coefficients $c_{11}^{(k)}, c_{12}^{(k)}, c_{13}^{(k)}, c_{33}^{(k)}, c_{44}^{(k)}(k=2,3)$ of the core vary exponentially through the thickness from the $c_{i j}^{f}$ value at the face sheet/core interface to the $c_{i j}^{c}$ value at the mid-plane according to

$$
c_{i j}^{(k)}\left(x_{3}\right)=c_{i j}^{c} \exp \left(\alpha^{(k)} \frac{x_{3}}{h}\right)
$$

where $\alpha^{(k)}$ can be determined in terms of elastic coefficients at the centre of the core and at the face sheet/core interfaces as

$$
\alpha^{(k)}=(-1)^{k} \frac{h}{h_{\mathrm{c}}} \ln \frac{c_{i j}^{c}}{c_{i j}^{f}}, \quad k=2,3
$$

All layers are assumed to be perfectly bonded together so that the continuity of stresses and displacements exists at all interfaces, i.e.

$$
\begin{aligned}
& x_{3}=x_{3}^{(1)}: \quad \sigma_{i 3}^{(2)}-\sigma_{i 3}^{(1)}=0, \quad u_{i}^{(2)}-u_{i}^{(1)}=0, \quad i=1,2,3 \\
& x_{3}=x_{3}^{(2)}: \quad \sigma_{i 3}^{(3)}-\sigma_{i 3}^{(2)}=0, \quad u_{i}^{(3)}-u_{i}^{(2)}=0, \quad i=1,2,3 \\
& x_{3}=x_{3}^{(3)}: \quad \sigma_{i 3}^{(4)}-\sigma_{i 3}^{(3)}=0, \quad u_{i}^{(4)}-u_{i}^{(3)}=0, \quad i=1,2,3
\end{aligned}
$$

where $u_{i}^{(k)}(k=1, \ldots, 4)$ are the components of displacement vector. The panel is subjected to transverse loading $Q$ on the top surface, so that

$$
x_{3}=h: \quad \sigma_{33}^{(4)}=Q\left(x_{1}, x_{2}\right), \quad \sigma_{13}^{(4)}=\sigma_{23}^{(4)}=0 .
$$

The loading $Q\left(x_{1}, x_{2}\right)$ is assumed to allow expansion into a double Fourier series

$$
Q\left(x_{1}, x_{2}\right)=-\sum_{m, n=1}^{\infty} q_{m n} \sin \frac{\pi m x_{1}}{a} \sin \frac{\pi n x_{2}}{b}
$$

where $q_{m n}$ is the intensity of the loading at the centre of the panel and $m$ and $n$ are wave numbers. The bottom surface of the panel is assumed to be load-free, i.e.

$$
x_{3}=0: \quad \sigma_{33}^{(1)}=\sigma_{13}^{(1)}=\sigma_{23}^{(1)}=0
$$

The Navier-type boundary conditions are assumed at the edges, so that

$$
\begin{aligned}
& x_{1}=0, a: \quad \sigma_{11}^{(k)}=0, \quad u_{2}^{(k)}=u_{3}^{(k)}=0, \quad k=1, \ldots, 4 \\
& x_{2}=0, b: \quad \sigma_{22}^{(k)}=0, \quad u_{1}^{(k)}=u_{3}^{(k)}=0, \quad k=1, \ldots, 4
\end{aligned}
$$

The boundary conditions, Eqs. (8), are representative of roller supports and analogous to simply supported edges in the plate theories [16]. 


\section{Solution using displacement potentials method}

To determine stresses and displacements in a sandwich panel subject to boundary conditions, Eqs. (5)-(8), two displacement potentials proposed by Kashtalyan and Rushchitsky [18] are employed. The displacements in each layer of the sandwich panel (the superscript indicating layer number is omitted henceforth for the sake of simplicity) can be expressed in terms of displacement potentials as

$$
\begin{aligned}
& u_{1}=\frac{\partial \Phi}{\partial x_{2}}-\left[\frac{c_{13}}{c_{11} c_{33}-c_{13}^{2}} \Delta_{2}-\frac{c_{33}}{c_{11} c_{33}-c_{13}^{2}} \frac{\partial^{2}}{\partial x_{3}^{2}}\right] \frac{\partial \Psi}{\partial x_{1}} \\
& u_{2}=-\frac{\partial \Phi}{\partial x_{1}}-\left[\frac{c_{13}}{c_{11} c_{33}-c_{13}^{2}} \Delta_{2}-\frac{c_{33}}{c_{11} c_{33}-c_{13}^{2}} \frac{\partial^{2}}{\partial x_{3}^{2}}\right] \frac{\partial \Psi}{\partial x_{2}} \\
& u_{3}=-\frac{1}{c_{44}} \Delta_{2} \frac{\partial \Psi}{\partial x_{3}}+\frac{\partial}{\partial x_{3}}\left[\frac{c_{13}}{c_{11} c_{33}-c_{13}^{2}} \Delta_{2}-\frac{c_{33}}{c_{11} c_{33}-c_{13}^{2}} \frac{\partial^{2}}{\partial x_{3}^{2}}\right] \Psi
\end{aligned}
$$

The components of the stress tensor can be expressed in terms of functions $\Phi$ and $\Psi$ as

$$
\begin{aligned}
& \sigma_{11}=\left[\left(\frac{c_{11} c_{13}-c_{12} c_{13}}{c_{11} c_{33}-c_{13}^{2}} \Delta_{2}+\frac{c_{12} c_{33}-c_{13}^{2}}{c_{11} c_{33}-c_{13}^{2}} \frac{\partial^{2}}{\partial x_{3}^{2}}\right) \frac{\partial^{2}}{\partial x_{2}^{2}}+\frac{\partial^{4}}{\partial x_{1}^{2} \partial x_{3}^{2}}\right] \Psi+\left(c_{11}-c_{12}\right) \frac{\partial^{2} \Phi}{\partial x_{1} \partial x_{2}} \\
& \sigma_{22}=\left[\left(\frac{c_{11} c_{13}-c_{12} c_{13}}{c_{11} c_{33}-c_{13}^{2}} \Delta_{2}+\frac{c_{12} c_{33}-c_{13}^{2}}{c_{11} c_{33}-c_{13}^{2}} \frac{\partial^{2}}{\partial x_{3}^{2}}\right) \frac{\partial^{2}}{\partial x_{1}^{2}}+\frac{\partial^{4}}{\partial x_{2}^{2} \partial x_{3}^{2}}\right] \Psi-\left(c_{11}-c_{12}\right) \frac{\partial^{2} \Phi}{\partial x_{1} \partial x_{2}} \\
& \sigma_{33}=\Delta_{2} \Delta_{2} \Psi \\
& \sigma_{12}=\frac{c_{12}-c_{11}}{c_{11} c_{33}-c_{13}^{2}}\left(c_{13} \Delta_{2}-c_{33} \frac{\partial^{2}}{\partial x_{3}^{2}}\right) \frac{\partial^{2} \Psi}{\partial x_{1} \partial x_{2}}-\frac{c_{11}-c_{12}}{2}\left(\frac{\partial^{2}}{\partial x_{1}^{2}}-\frac{\partial^{2}}{\partial x_{2}^{2}}\right) \Phi \\
& \sigma_{13}=-\Delta_{2} \frac{\partial^{2} \Psi}{\partial x_{1} \partial x_{3}}+c_{44} \frac{\partial^{2} \Phi}{\partial x_{2} \partial x_{3}} \\
& \sigma_{23}=-\Delta_{2} \frac{\partial^{2} \Psi}{\partial x_{2} \partial x_{3}}-c_{44} \frac{\partial^{2} \Phi}{\partial x_{1} \partial x_{3}}
\end{aligned}
$$

where

$$
\Delta_{2}=\frac{\partial^{2}}{\partial x_{1}^{2}}+\frac{\partial^{2}}{\partial x_{2}^{2}}
$$

Functions $\Phi$ and $\Psi$ satisfy the following differential equations [18]

$$
\begin{aligned}
& \tilde{m}\left(x_{3}\right) \Delta_{2} \Phi+g^{0} \frac{\partial}{\partial x_{3}}\left[\tilde{m}\left(x_{3}\right) \frac{\partial \Phi}{\partial x_{3}}\right]=0 \\
& c_{11}^{0} \Delta_{2} \Delta_{2} \Psi-c_{13}^{0} \Delta_{2} \frac{\partial^{2} \Psi}{\partial x_{3}^{2}}+\left(c_{11}^{0} c_{33}^{0}-c_{13}^{02}\right) \tilde{m}\left(x_{3}\right) \\
& \quad \times\left\{\frac{1}{c_{44}^{0}} \frac{\partial}{\partial x_{3}}\left[\tilde{m}^{-1}\left(x_{3}\right) \Delta_{2} \frac{\partial \Psi}{\partial x_{3}}\right]+\frac{c_{33}^{0}}{c_{11}^{0} c_{33}^{0}-c_{13}^{02}} \frac{\partial^{2}}{\partial x_{3}}\left[\tilde{m}^{-1}\left(x_{3}\right) \frac{\partial^{2} \Psi}{\partial x_{3}^{2}}\right]\right. \\
& \left.\quad-\frac{c_{13}^{0}}{c_{11}^{0} c_{33}^{0}-c_{13}^{0}} \frac{\partial^{2}}{\partial x_{3}}\left[\tilde{m}^{-1}\left(x_{3}\right) \Delta_{2} \Psi\right]\right\}=0
\end{aligned}
$$

where

$$
g^{0}=\frac{2 c_{44}}{c_{11}-c_{12}}=\frac{2 c_{44}^{0}}{c_{11}^{0}-c_{12}^{0}}=\text { const }
$$

subject to boundary conditions (5)-(8). Constant $g^{0}$, given by (10c), represents the ratio between the shear moduli in a plane of isotropy and a plane normal to it. For isotropic materials, it is equal to unity, for transversely isotropic materials it can be used to characterise the degree of anisotropy exhibited by a material. 
Solution of Eq. (10) starts with separating variables in the displacement functions in the form

$$
\begin{aligned}
& \Phi\left(x_{1}, x_{2}, x_{3}\right)=\widehat{\widehat{\Phi}}\left(x_{1}, x_{2}\right) \widehat{\Phi}\left(x_{3}\right) \\
& \Psi\left(x_{1}, x_{2}, x_{3}\right)=\widehat{\widehat{\Psi}}\left(x_{1}, x_{2}\right) \widehat{\Psi}\left(x_{3}\right)
\end{aligned}
$$

Substitution of these expressions into Eqs. (10a) and (10b) allows the following four differential equations to be derived

$$
\begin{gathered}
\Delta_{2} \hat{\bar{\Phi}}+k_{\Phi}^{2} \widehat{\widehat{\Phi}}=0 \\
\Delta_{2} \widehat{\widehat{\Psi}}+k_{\Psi}^{2} \widehat{\widehat{\Psi}}=0 \\
{\left[\frac{d^{2}}{d x_{3}^{2}}+\frac{\tilde{m^{\prime}}\left(x_{3}\right)}{\tilde{m}\left(x_{3}\right)} \frac{d}{d x_{3}}-\frac{k_{\Phi}^{2}}{g^{0}}\right] \widehat{\Phi}=0} \\
\tilde{m}\left(x_{3}\right) \frac{d^{2}}{d x_{3}}\left[\tilde{m}^{-1}\left(x_{3}\right) \frac{d^{2} \widehat{\Psi}}{d x_{3}^{2}}\right]+\frac{c_{13}^{0}}{c_{33}^{0}} k_{\Psi}^{2} \tilde{m}\left(x_{3}\right) \frac{d^{2}}{d x_{3}^{2}}\left[\tilde{m}^{-1}\left(x_{3}\right) \widehat{\Psi}\right] \\
-\frac{c_{11}^{0} c_{33}^{0}-c_{13}^{0^{2}}}{c_{33}^{0} c_{44}^{0}} k_{\Psi}^{2} \tilde{m}\left(x_{3}\right) \frac{d}{d x_{3}}\left[\tilde{m}^{-1}\left(x_{3}\right) \frac{d \Psi}{d x_{3}}\right]+\frac{c_{13}^{0}}{c_{33}^{0}} k_{\Psi}^{2} \frac{d^{2} \widehat{\Psi}}{d x_{3}^{2}}+\frac{c_{11}^{0}}{c_{33}^{0}} k_{\Psi}^{4} \widehat{\Psi}=0
\end{gathered}
$$

For a simply supported plate subjected to sinusoidal loading, with the boundary conditions described by Eqs. (5)-(8), functions $\widehat{\widehat{\Phi}}=\widehat{\widehat{\Phi}}\left(x_{1}, x_{2}\right)$ and $\widehat{\widehat{\Psi}}=\widehat{\widehat{\Psi}}\left(x_{1}, x_{2}\right)$ can be chosen as

$$
\begin{aligned}
& \widehat{\widehat{\Phi}}\left(x_{1}, x_{2}\right)=\cos \frac{\pi m x_{1}}{a} \cos \frac{\pi n x_{2}}{b} \\
& \widehat{\bar{\Psi}}\left(x_{1}, x_{2}\right)=\sin \frac{\pi m x_{1}}{a} \sin \frac{\pi n x_{2}}{b}
\end{aligned}
$$

Then the boundary conditions on the edges of the plate are satisfied exactly.

Selecting the inhomogeneity function such that it is an exponential one, Eqs. (3) and (4), reduces Eqs. (12c) and (12d) to the following second- and fourth-order differential equations with constant coefficients

$$
\begin{gathered}
h^{2} \frac{d^{2} \widehat{\Phi}}{d x_{3}^{2}}+\alpha h \frac{d \widehat{\Phi}}{d x_{3}}-\frac{k_{\Phi}^{2} h^{2}}{g^{0}} \widehat{\Phi}=0 \\
h^{4} \frac{d^{4} \widehat{\Psi}}{d x_{3}^{4}}-2 \alpha h^{3} \frac{d^{3} \widehat{\Psi}}{d x_{3}^{3}}+h^{2}\left[\alpha^{2}+\left(2 \frac{c_{13}^{0}}{c_{33}^{0}}-\frac{c_{11}^{0} c_{33}^{0}-c_{13}^{02}}{c_{33}^{0} c_{44}^{0}}\right) k_{\Psi}^{2} h^{2}\right] \frac{d^{2} \widehat{\Psi}}{d x_{3}^{2}} \\
-\alpha k_{\Psi}^{2} h^{3}\left[2 \frac{c_{13}^{0}}{c_{33}^{0}}-\frac{c_{11}^{0} c_{33}^{0}-c_{13}^{02}}{c_{33}^{0} c_{44}^{0}}\right] \frac{d \widehat{\Psi}}{d x_{3}}+\frac{k_{\Psi}^{2} h^{2}\left(c_{11}^{0} k_{\Psi}^{2} h^{2}+c_{13}^{0} \alpha^{2}\right)}{c_{33}^{0}} \widehat{\Psi}=0
\end{gathered}
$$

where

$$
k_{\Phi}^{2}=k_{\Psi}^{2}=\pi^{2}\left[\left(\frac{m}{a}\right)^{2}+\left(\frac{n}{b}\right)^{2}\right]
$$

It is worth mentioning that the exponential variation of material properties with transverse co-ordinate used in the above solution is not as restrictive as it may seem since any variation of material properties through the thickness can be handled using a piecewise-exponential model proposed in Woodward and Kashtalyan [34] .

Solutions to Eqs. (14a) and (14b) will vary depending on the values of the elastic constants and parameters $k_{\Phi}$ and $k_{\Psi}$, as detailed in Woodward and Kashtalyan [32].

For example, if the discriminant of the characteristic equation corresponding to Eq. (14b) is negative, then

$$
\begin{aligned}
\widehat{\Psi}= & h^{4} \exp \left(\frac{\alpha x_{3}}{2 h}\right)\left[A_{1} \cosh \frac{\lambda x_{3}}{h} \cos \frac{\mu x_{3}}{h}+A_{2} \cosh \frac{\lambda x_{3}}{h} \sin \frac{\mu x_{3}}{h}\right. \\
& \left.+A_{3} \sinh \frac{\lambda x_{3}}{h} \cos \frac{\mu x_{3}}{h}+A_{4} \sinh \frac{\lambda x_{3}}{h} \sin \frac{\mu x_{3}}{h}\right]
\end{aligned}
$$


where $\lambda$ and $\mu$ are

$$
\begin{aligned}
\lambda=\frac{1}{4} & {\left[8\left(-\frac{c_{13}^{0}}{c_{33}^{0}}+\frac{1}{2} \frac{c_{11}^{0}}{c_{44}^{0}}-\frac{1}{2} \frac{c_{13}^{0}}{c_{33}^{0} c_{44}^{0}}\right) k_{\Psi}^{2} h^{2}+2 \alpha^{2}+4\left(2 \alpha^{2} h^{2} k_{\Psi}^{2} \frac{c_{13}^{0}}{c_{33}^{0}}+\alpha^{2} h^{2} k_{\Psi}^{2} \frac{c_{11}^{0}}{c_{44}^{0}}\right.\right.} \\
& \left.\left.-\alpha^{2} h^{2} k_{\Psi}^{2} \frac{c_{13}^{0^{2}}}{c_{33}^{0} c_{44}^{0}}+4 h^{4} k_{\Psi}^{4} \frac{c_{13}^{0}}{c_{33}^{0}}+\frac{1}{4} \alpha^{4}\right)^{\frac{1}{2}}\right]^{\frac{1}{2}}
\end{aligned}
$$

and

$$
\begin{aligned}
\mu=\frac{1}{4} & {\left[8\left(\frac{c_{13}^{0}}{c_{33}^{0}}-\frac{1}{2} \frac{c_{11}^{0}}{c_{44}^{0}}+\frac{1}{2} \frac{c_{13}^{0^{2}}}{c_{33}^{0} c_{44}^{0}}\right) k_{\Psi}^{2} h^{2}-2 \alpha^{2}+4\left(2 \alpha^{2} h^{2} k_{\Psi}^{2} \frac{c_{13}^{0}}{c_{33}^{0}}+\alpha^{2} h^{2} k_{\Psi}^{2} \frac{c_{11}^{0}}{c_{44}^{0}}\right.\right.} \\
& \left.\left.-\alpha^{2} h^{2} k_{\Psi}^{2} \frac{c_{13}^{0^{2}}}{c_{33}^{0} c_{44}^{0}}+4 h^{4} k_{\Psi}^{4} \frac{c_{13}^{0}}{c_{33}^{0}}+\frac{1}{4} \alpha^{4}\right)^{\frac{1}{2}}\right]^{\frac{1}{2}}
\end{aligned}
$$

The solution of second-order equation (14a) yields

$$
\widehat{\Phi}=\frac{h^{2}}{c_{44}^{0}} \exp \left(-\frac{\alpha x_{3}}{2 h}\right)\left[A_{5} \cosh \left(\frac{\beta x_{3}}{h}\right)+A_{6} \sinh \left(\frac{\beta x_{3}}{h}\right)\right]
$$

where

$$
\beta=\sqrt{\frac{\alpha^{2}}{4}+k_{\Phi}^{2} h^{2} \frac{c_{11}^{0}-c_{12}^{0}}{c_{44}^{0}}}
$$

In Eqs. (15)-(16), $A_{i}(i=1, \ldots, 6)$ are arbitrary constants.

Substitution of functions $\widehat{\bar{\Phi}}$ and $\widehat{\bar{\Psi}}$, Eqs. (13a, 13b), and functions $\widehat{\Phi}$ and $\widehat{\Psi}$, Eqs. (15, 16), into Eqs. (11a, 11b) and then into Eq. (9), gives the following expressions for stresses and displacements in a simply supported sandwich panel under sinusoidal loading, with transversely isotropic functionally graded core having exponential dependence of the elastic constants on the transverse co-ordinate

$$
\begin{aligned}
& u_{1}^{(k)}=\sum_{m, n=1}^{\infty} \sum_{j=1}^{6} A_{m n j}^{(k)} U_{1 j}^{(k)}\left(m, n, x_{3}\right) \cos \frac{\pi m x_{1}}{a} \sin \frac{\pi n x_{2}}{b} \\
& u_{2}^{(k)}=\sum_{m, n=1}^{\infty} \sum_{j=1}^{6} A_{m n j}^{(k)} U_{2 j}^{(k)}\left(m, n, x_{3}\right) \sin \frac{\pi m x_{1}}{a} \cos \frac{\pi n x_{2}}{b} \\
& u_{3}^{(k)}=\sum_{m, n=1}^{\infty} \sum_{j=1}^{6} A_{m n j}^{(k)} U_{3 j}^{(k)}\left(m, n, x_{3}\right) \sin \frac{\pi m x_{1}}{a} \sin \frac{\pi n x_{2}}{b} \\
& \sigma_{11}^{(k)}=\sum_{m, n=1}^{\infty} \sum_{j=1}^{6} A_{m n j}^{(k)} P_{11 j}^{(k)}\left(m, n, x_{3}\right) \sin \frac{\pi m x_{1}}{a} \sin \frac{\pi n x_{2}}{b} \\
& \sigma_{22}^{(k)}=\sum_{m, n=1}^{\infty} \sum_{j=1}^{6} A_{m n j}^{(k)} P_{22 j}^{(k)}\left(m, n, x_{3}\right) \sin \frac{\pi m x_{1}}{a} \sin \frac{\pi n x_{2}}{b} \\
& \sigma_{33}^{(k)}=\sum_{m, n=1}^{\infty} \sum_{j=1}^{6} A_{m n j}^{(k)} P_{33 j}^{(k)}\left(m, n, x_{3}\right) \sin \frac{\pi m x_{1}}{a} \sin \frac{\pi n x_{2}}{b} \\
& \sigma_{12}^{(k)}=\sum_{m, n=1}^{\infty} \sum_{j=1}^{6} A_{m n j}^{(k)} P_{12 j}^{(k)}\left(m, n, x_{3}\right) \cos \frac{\pi m x_{1}}{a} \cos \frac{\pi n x_{2}}{b}
\end{aligned}
$$




$$
\begin{aligned}
\sigma_{13}^{(k)} & =\sum_{m, n=1}^{\infty} \sum_{j=1}^{6} A_{m n j}^{(k)} P_{13 j}^{(k)}\left(m, n, x_{3}\right) \cos \frac{\pi m x_{1}}{a} \sin \frac{\pi n x_{2}}{b} \\
\sigma_{23}^{(k)} & =\sum_{m, n=1}^{\infty} \sum_{j=1}^{6} A_{m n j}^{(k)} P_{23 j}^{(k)}\left(m, n, x_{3}\right) \sin \frac{\pi m x_{1}}{a} \cos \frac{\pi n x_{2}}{b}
\end{aligned}
$$

Functions $U_{i j}^{(k)}$ and $P_{r t j}^{(k)}$ are specified in "Appendix".

Substitution of Eqs. (17)-(18) into the stress and displacement continuity conditions at the interfaces $x_{3}=x_{3}^{(k)}(k=1, \ldots 4)$, Eq. (5), and boundary conditions, at the top $\left(x_{3}=h\right)$ and bottom $\left(x_{3}=-h\right)$ surfaces of the panel, Eqs. (6)-(7), produces a set of 24 linear algebraic equations with respect to arbitrary constants $A_{m n j}^{(k)}$ for any combination of $m$ and $n$. These are solved in Matlab using LU decomposition method. Boundary conditions at the edges of the panel, Eqs. (8a, 8b), are satisfied exactly.

\section{Validation}

Since isotropy is a particular case of transverse isotropy, the proposed solution for a sandwich panel with transversely isotropic graded core can be used to obtain the solution for the sandwich panel with isotropic graded core if the elastic coefficients are adjusted as follows

$$
c_{11}=c_{33}=\frac{E(1-v)}{(1+v)(1-2 v)}, \quad c_{12}=c_{13}=\frac{E v}{(1+v)(1-2 v)}, \quad c_{44}=G, \quad G=\frac{E}{2(1+v)}
$$

with

$$
\frac{c_{11} c_{13}-c_{12} c_{13}}{c_{11} c_{33}-c_{13}^{2}}=\frac{c_{12} c_{33}-c_{13}^{2}}{c_{11} c_{33}-c_{13}^{2}}=v, \quad c_{11}-c_{12}=2 G
$$

It was shown by Kashtalyan and Rushchitsky [18] that if for graded transversely isotropic material the elastic coefficients are adjusted as $(18 \mathrm{a}, 18 \mathrm{~b})$, the representation of displacements and stresses in terms of displacement potential functions $\Phi$ and $\Psi(9 \mathrm{a}-9 \mathrm{j}, 10 \mathrm{a}-10 \mathrm{c})$ fully coincides with that obtained by Plevako [22] for inhomogeneous isotropic material. Whilst for homogeneous materials transition from transverse isotropy to isotropy is associated with the roots the characteristic equation changing type (from distinct roots for transversely isotropic materials to multiple roots for isotropic materials), this is not the case when the material is graded.

Table 1 shows numerical results for the normalised displacements $\bar{u}_{i}=\frac{c_{44}^{0} u_{i}}{q_{11} h}$ and normalised stresses $\bar{\sigma}_{i j}=\frac{\sigma_{i j}}{q_{11}}$ in a square sandwich panel $(a / h=b / h=3)$ obtained using the transversely isotopic solution, Eqs. (18), and the isotropic solution [17] which employed Plevako's representation. It can be seen that they are in exact agreement with each other.

In order to verify that the solution presented in the previous section is correctly modelling transversely isotropic materials, comparison is also made with a transversely isotropic homogenous plate using two independent models: firstly, an analytical solution for a homogenous transversely isotropic plate obtained using Elliot's displacement functions [13] and, secondly, a finite element model for a homogeneous plate that was set up in a relatively straightforward manner in ABAQUS.

Table 1 Normalised displacements and stresses in a square isotropic graded plate using 'isotropic' and 'transversely isotropic' solutions

\begin{tabular}{lccc}
\hline & Isotropic solution [17] & Transversely isotropic solution (present paper) & Difference (\%) \\
\hline$\frac{c_{44}^{0} u_{3}}{q_{11} h}(0.5 a, 0.5 b, 0.5 h)$ & -4.29778 & -4.29778 & 0.0 \\
$\frac{\sigma_{11}}{q_{11}}(0.5 a, 0.5 b, 0.5 h)$ & 0.653339 & 0.653339 & 0.0 \\
$\frac{\sigma_{12}}{q_{11}}(0,0,0.5 h)$ & -0.43007 & -0.43007 & 0.0 \\
$\frac{\sigma_{13}}{q_{11}}(0,0.5 b, 0.5 h)$ & -0.64606 & -0.64606 & 0.0 \\
\hline
\end{tabular}




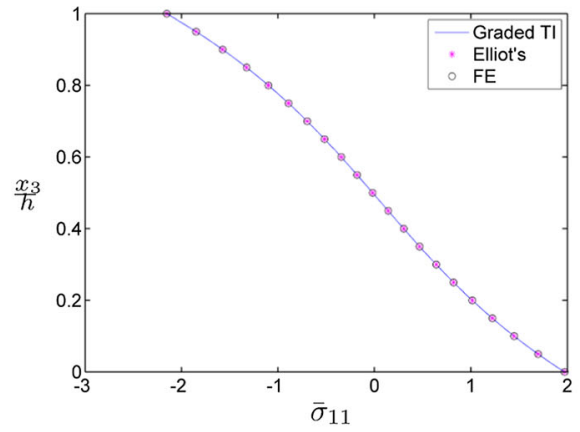

(a)

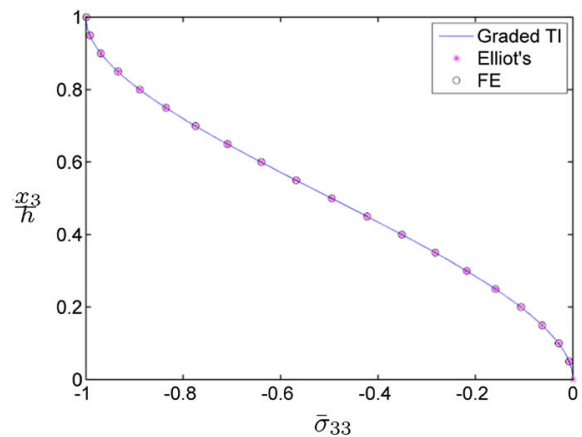

(c)

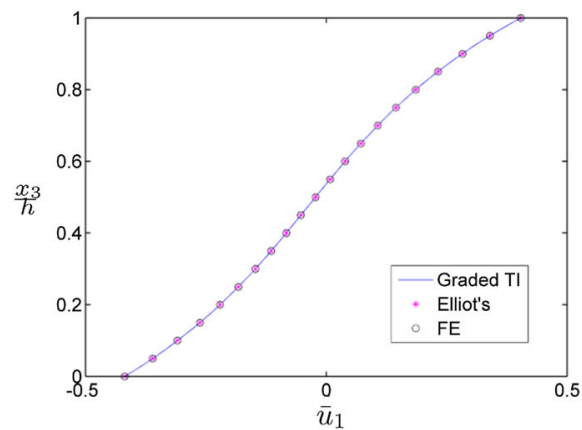

(e)

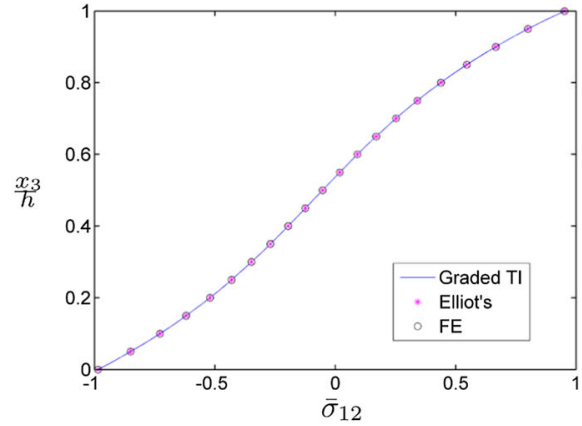

(b)

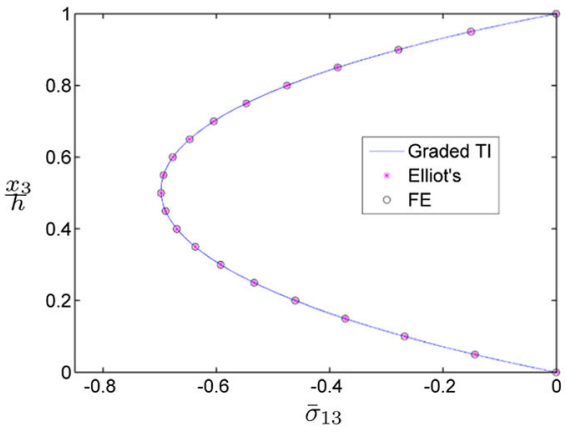

(d)

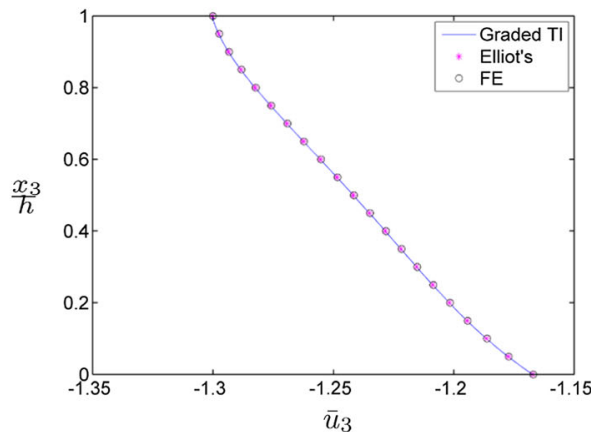

(f)

Fig. 2 Through-thickness variation of the normalised stresses and displacements in a transversely isotropic homogeneous plate: a in-plane normal stress $\bar{\sigma}_{11}\left(0.5 a, 0.5 b, x_{3}\right)$; b in-plane shear stress $\bar{\sigma}_{12}\left(0,0, x_{3}\right)$; $\mathbf{c}$ out-of-plane normal stress $\bar{\sigma}_{33}\left(0.5 a, 0.5 b, x_{3}\right)$; d transverse shear stress $\bar{\sigma}_{13}\left(0,0.5 b, x_{3}\right)$; e in-plane displacement $\bar{u}_{1}\left(0,0.5 b, x_{3}\right)$; f transverse displacement $\bar{u}_{3}\left(0.5 a, 0.5 b, x_{3}\right)$

Table 2 Elastic constants for alumina

\begin{tabular}{llllll}
\hline$c_{11}(\mathrm{GPa})$ & $c_{12}(\mathrm{GPa})$ & $c_{13}(\mathrm{GPa})$ & $c_{33}(\mathrm{GPa})$ & $c_{44}(\mathrm{GPa})$ & $g^{0}$ \\
\hline 460.2 & 174.7 & 127.4 & 509.5 & 126.9 & 0.889 \\
\hline
\end{tabular}

The results for stresses and displacements in a homogenous, transversely isotropic plate are shown in Fig. 2. Through-thickness variation of the normalised stresses $\bar{\sigma}_{i j}=\sigma_{i j} / q_{11}$ and normalised displacements $\bar{u}_{i}=\frac{c_{41}^{0} u_{i}}{q_{11} h}$ in a thick square plate with $a / h=b / h=3$ is predicted by the three different models: Graded Transversely Isotropic (Graded TI); Elliot's; Finite Element method (FE). Calculations are performed for alumina, with material properties [13] listed in Table 2.

Excellent agreement is found between all three models. In order to model a homogenous transversely isotropic plate, parameter $\alpha$ is set sufficiently close to zero when defining the inhomogeneity function, Eq. (3). 
Table 3 Engineering constants of model materials

\begin{tabular}{lllcrll}
\hline Materials & $E_{p}(\mathrm{GPa})$ & $E_{t}(\mathrm{GPa})$ & $G_{t}(\mathrm{GPa})$ & $v_{p}$ & $v_{p t}$ & $v_{t p}$ \\
\hline 'Base material' & 380.7 & 458.37 & 126.9 & 0.334 & 0.167 & 0.201 \\
'Low $G_{t}$ material' & 380.7 & 458.37 & 12.69 & 0.334 & 0.167 & 0.201 \\
'Very low $G_{t}$ material' & 380.7 & 458.37 & 2.54 & 0.334 & 0.167 & 0.201 \\
'Equivalent Isotropic material' & 380.7 & 380.7 & 142.7 & 0.334 & 0.334 & 0.334 \\
\hline
\end{tabular}

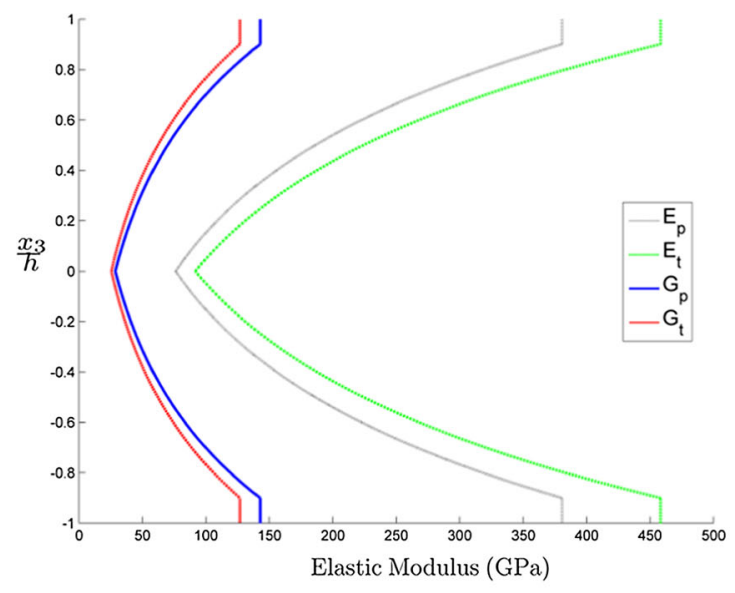

(a)

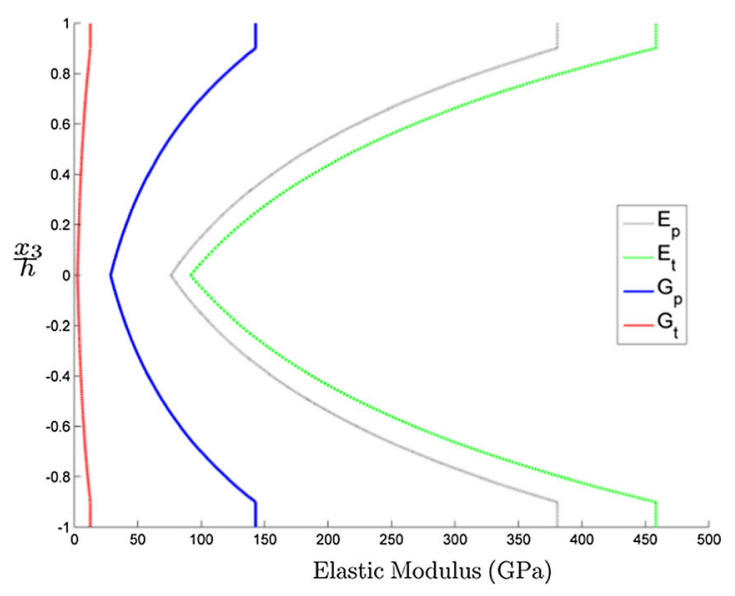

(b)

Fig. 3 Through-thickness variation of engineering constants: a 'base material'; b 'low $G_{t}$ material'

For alumina, the discriminant of characteristic equation was positive, and the characteristic equation had four real roots. In order to ensure that the solution was valid for any transversely isotropic materials, comparison was also made for materials with the other cases of roots, and excellent agreement was again found.

\section{Results and discussion}

In order to study behaviour of sandwich panels with a transversely isotropic graded core, a 'base' transversely isotropic model material was chosen, with properties of alumina listed in Table 2, and an elastic property gradient was then imposed in the transverse direction. The stiffness gradient between the face sheets and the centre of the core was taken as $\left(c_{i j}^{0}\right)_{\text {face }} /\left(c_{i j}^{0}\right)_{\text {core }}=5$, meaning that the face sheets were five times stiffer than the core centre. The thickness of the face sheets is taken as $h_{\mathrm{f}}=0.05 h_{0}$.

In order to understand combined effect of anisotropy and stiffness gradient, a comparison is made between the functionally graded 'base material', and a functionally graded transverse shear compliant material with lower transverse shear modulus ('low $G_{t}$ material'). Engineering properties of both materials are listed in Table 3, which shows values of engineering constants in the face sheets: Young's modulus in the plane of isotropy $E_{p}$; Young's modulus in the transverse direction $E_{t}$; shear modulus in the transverse direction $G_{t}$; Poisson's ratio in the plane of isotropy $v_{p}$; Poisson's ratios $v_{p t}$ and $v_{p t}$ are related as $\frac{v_{p t}}{E_{p}}=\frac{v_{t p}}{E_{t}}$. Additionally, 'very low $G_{t}$ material' and 'equivalent isotropic material' were considered. Variation of the engineering constants for the 'base material' and 'low $G_{t}$ material' is shown in Fig. 3.

\subsection{Transverse shear effects under distributed loading}

Effect of transverse shear modulus on the through-thickness variation of normalised stresses and displacements in a sandwich panel under one-term sinusoidal loading is shown in Fig. 4 for three transversely isotropic graded materials listed in Table 3.

From Fig. 4a, it can be seen that in all three panels, as expected, the upper face sheet is in compression and the lower face sheet is in tension. Considering the in-plane normal stresses $\bar{\sigma}_{11}$ in the panel core, it can be seen 


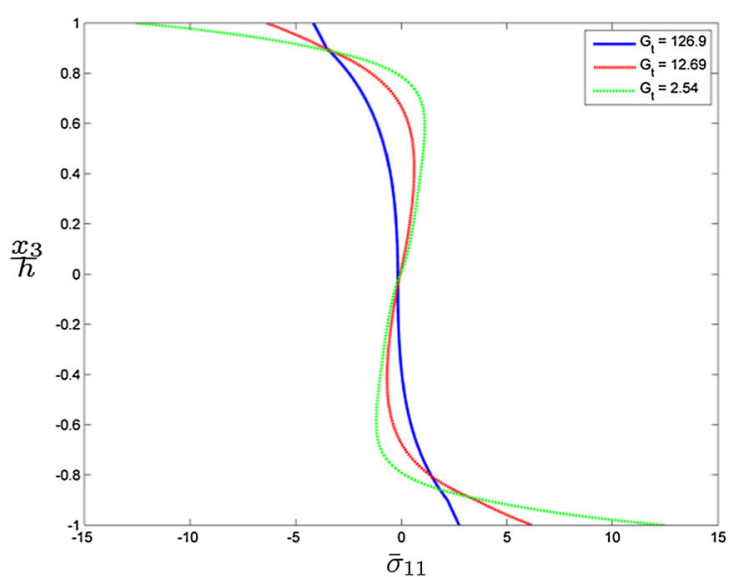

(a)

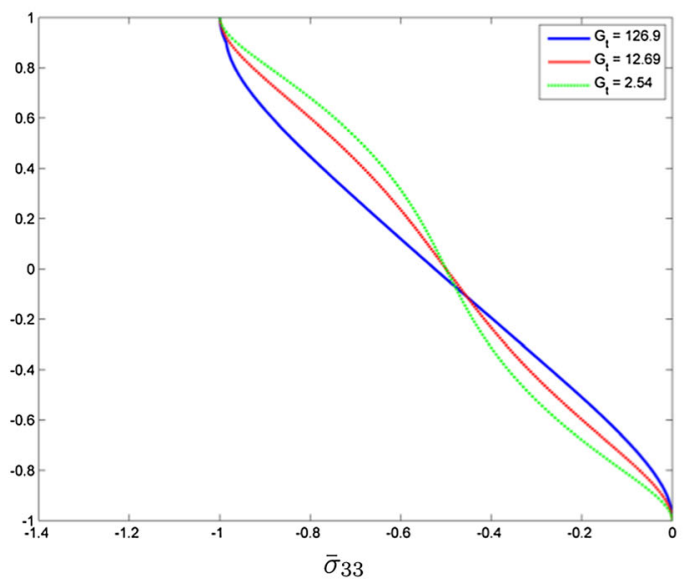

(c)

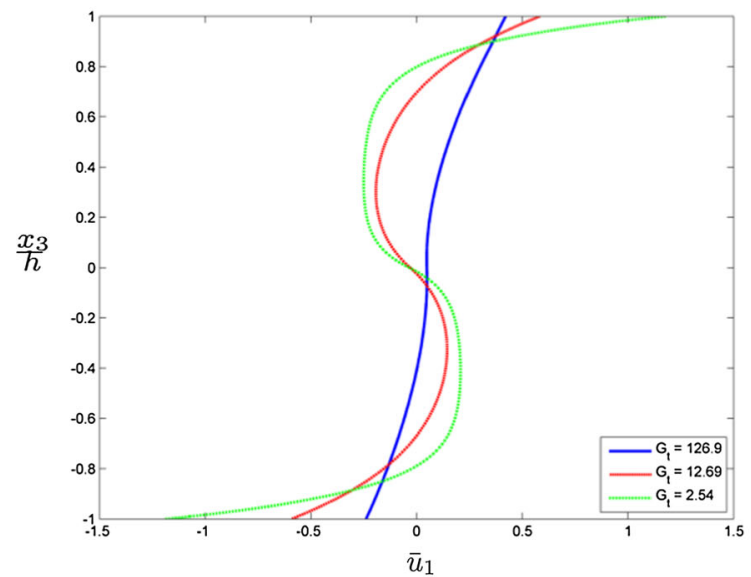

(e)

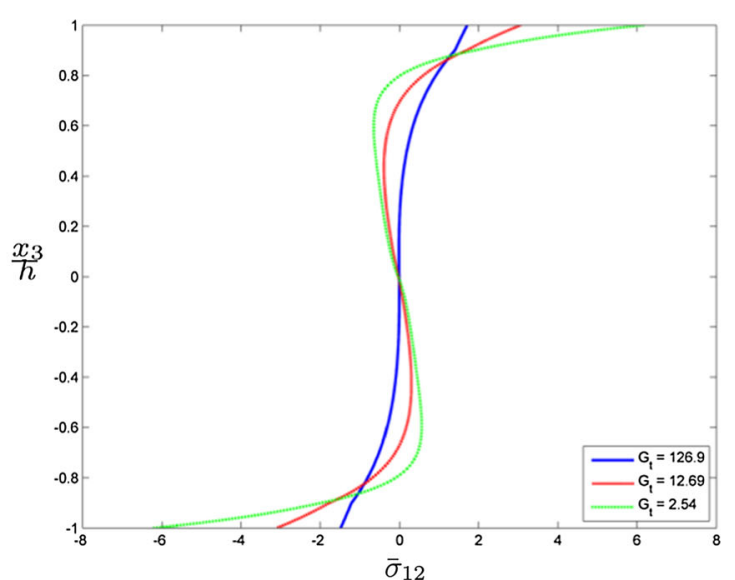

(b)

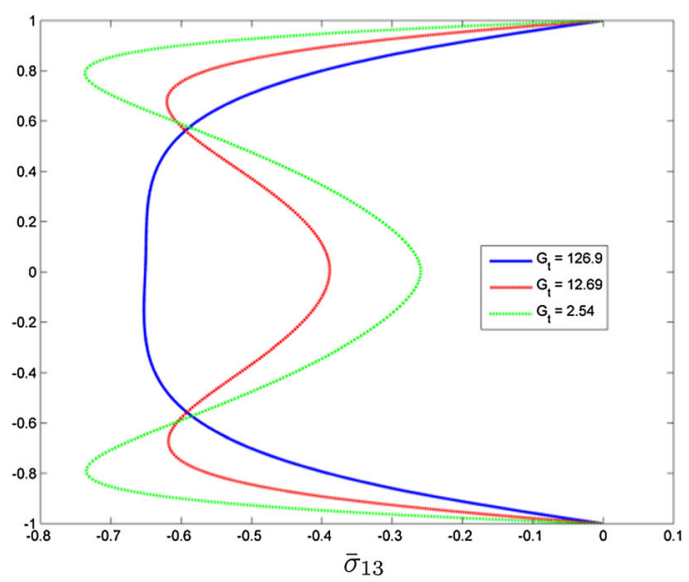

(d)

Fig. 4 Effect of transverse shear modulus on through-thickness variation of stresses and displacements in sandwich panels with transversely isotropic graded core: $\mathbf{a}$ in-plane normal stress $\bar{\sigma}_{11}\left(0.5 a, 0.5 b, x_{3}\right)$; b in-plane shear stress $\bar{\sigma}_{12}\left(0,0, x_{3}\right)$; c out-ofplane normal stress $\bar{\sigma}_{33}\left(0.5 a, 0.5 b, x_{3}\right) ; \mathbf{d}$ transverse shear stress $\bar{\sigma}_{13}\left(0,0.5 b, x_{3}\right)$; e in-plane displacement $\bar{u}_{1}\left(0,0.5 b, x_{3}\right)$ 


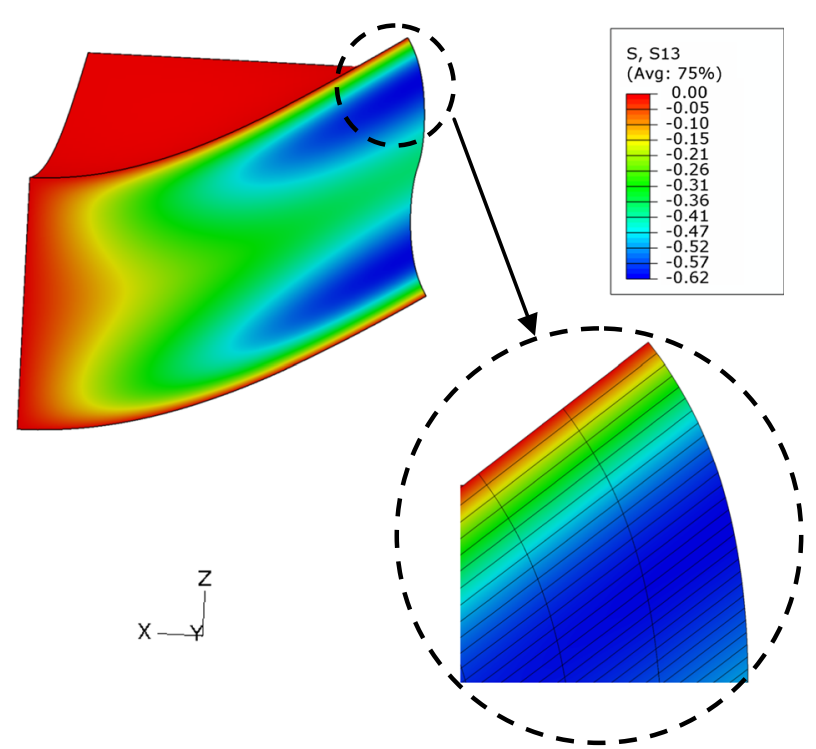

Fig. 5 Distribution of normalised transverse shear stress $\bar{\sigma}_{13}$ on one quarter of panel for 'low $G_{t}$ material'

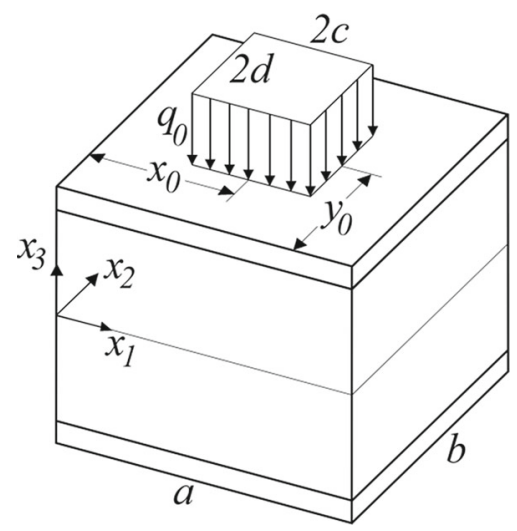

Fig. 6 Panel subjected to patch load relative to Cartesian coordinates

that in the panel with base value of transverse shear modulus, the in-plane normal stresses gradually increase (due to the functionally graded core properties) from zero at the core centre to a maximum at the outer edges of the face sheets. The sign of these stresses shows that the upper half of the core and upper face sheet is in compression, whilst the lower half of the core and lower face sheet is in tension. However, in the panels with low transverse shear modulus, this is not the case. In these panels, the upper half of the core can be seen to be in tension and the lower half in compression. As such all four layers of the panel are no longer bending as one; instead, they are working independently as plates in bending, meaning the panel is no longer behaving as a true sandwich panel.

For the panels with low transverse shear modulus, the effects of shear deformation can be also observed in Fig. 4d. The transverse shear stress no longer varies from a minimum at the outer edges of the face sheets to a maximum at the panel centre. Instead, the face sheets can be seen to be carrying a greater proportion of the shear stresses, whilst shear stresses at the centre of the core are reduced.

The effects of transverse shear modulus on the in-plane displacement are immediately obvious in Fig. 4e. For the panel with low transverse shear modulus, individual peaks in displacement are located in the upper and lower halves of the core and variation is highly nonlinear in relation to the mid-plane.

Figure 5 shows a snapshot from a finite element simulation of a panel with low core shear modulus under sinusoidal loading where this effect is detailed. 


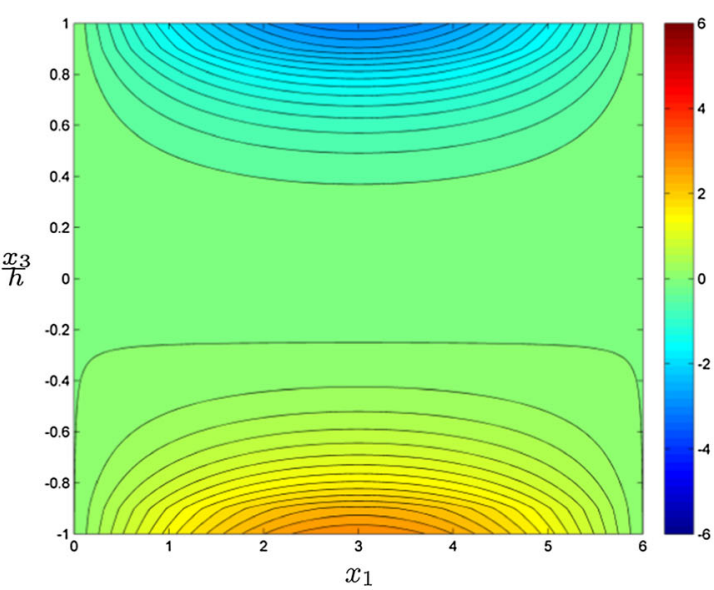

(a)

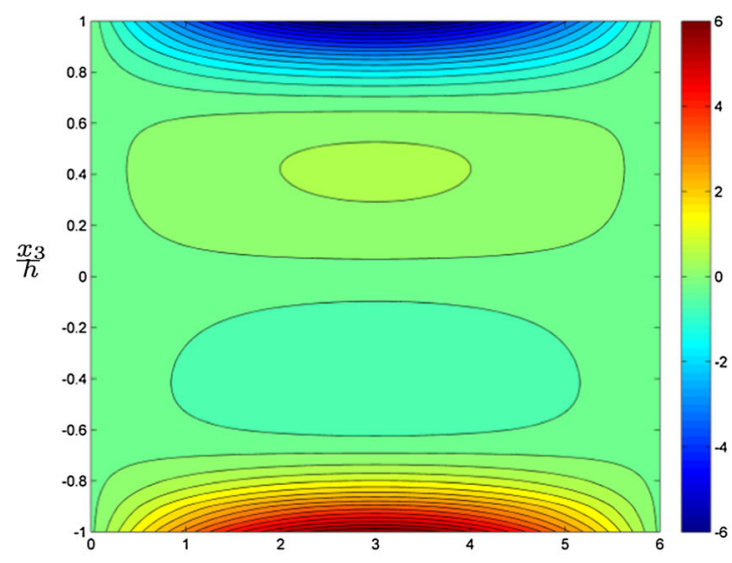

(c)

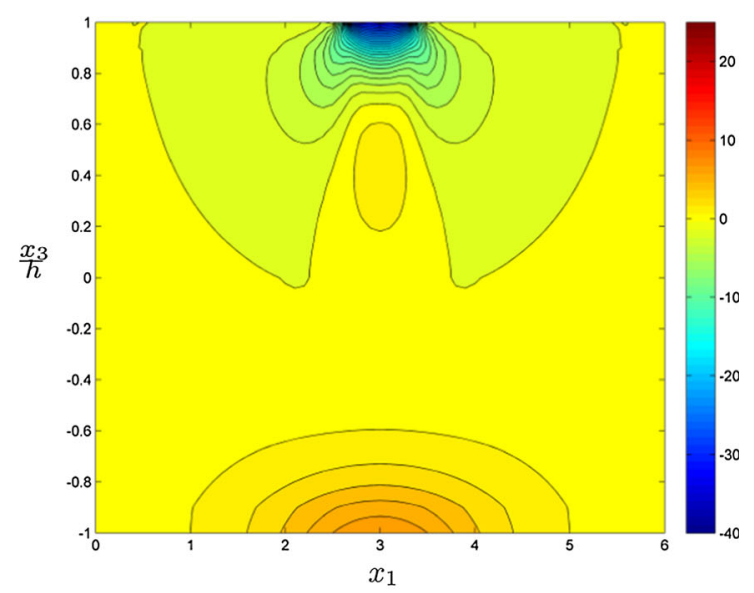

(b)

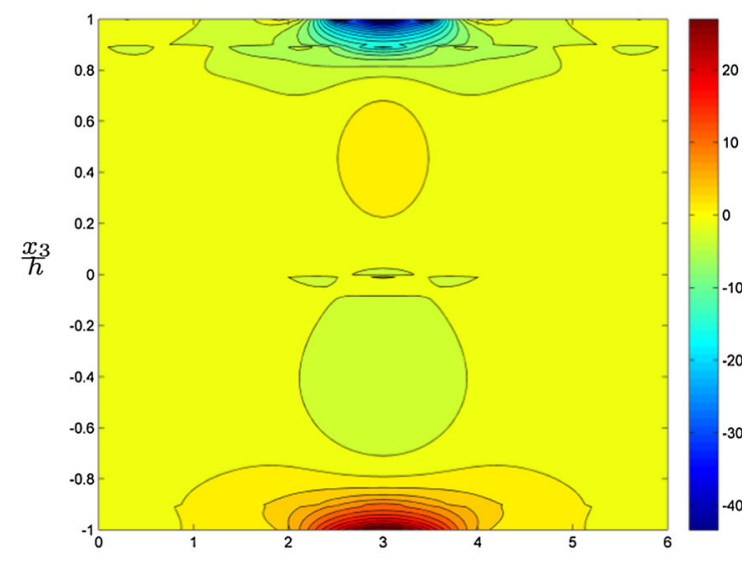

(d)

Fig. 7 Through-thickness variation of normalised in-plane normal stress $\bar{\sigma}_{11}$ under sinusoidal and patch loadings on a central vertical section $x_{2}=0.5 b$ : a 'base material' under sinusoidal loading; $\mathbf{b}$ 'base material' under patch loading; c 'low $G_{t}$ material' under sinusoidal loading; d 'low $G_{t}$ material' under patch loading

\subsection{Transverse shear effects under localised loading}

To investigate the effect of load localisation, two types of loading — sinusoidal loading and patch loading —are examined. Both are applied to thick panels with $a / h_{0}=b / h_{0}=3$.

Patch loading (Fig. 6) is defined as

$$
Q\left(x_{1}, x_{2}\right)= \begin{cases}q_{0}, & x_{0}-c<x_{1}<x_{0}+c, \quad y_{0}-d<x_{2}<y_{0}+d \\ 0, & \text { elsewhere }\end{cases}
$$

For patch loading, coefficients in the double Fourier series, Eq. (6b), are

$$
q_{m n}=\frac{16}{\pi^{2} m n} \sin \frac{\pi m x_{0}}{a} \sin \frac{\pi m y_{0}}{b} \sin \frac{\pi m c}{a} \sin \frac{\pi m d}{b}
$$

Patch loading is taken over an area with dimensions $c=d=a / 16$ and applied at the centre of the panel $\left(x_{0}=0.5 a, \quad y_{0}=0.5 b\right)$. In order for an accurate comparison to be made between panels subjected to different types of loading, the intensity of the patch load is adjusted so that the overall load remains the same as that applied in the sinusoidal case. For the considered panel and patch sizes, this requirement leads to $q_{0}^{\text {patch }}=25.94 q_{0}^{\text {sinusoidal }}$. 


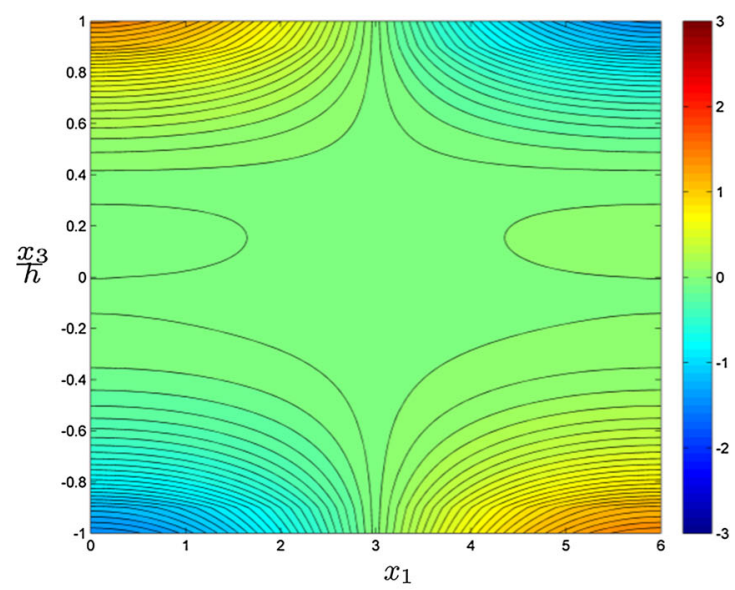

(a)

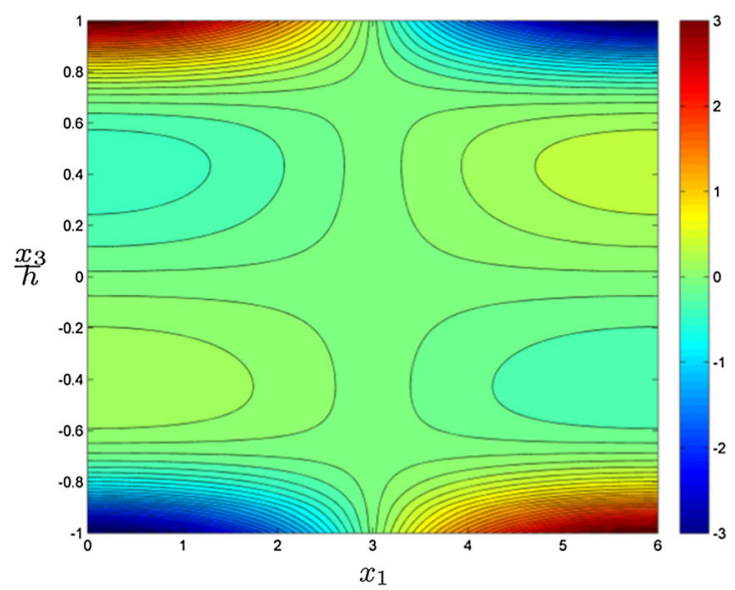

(c)

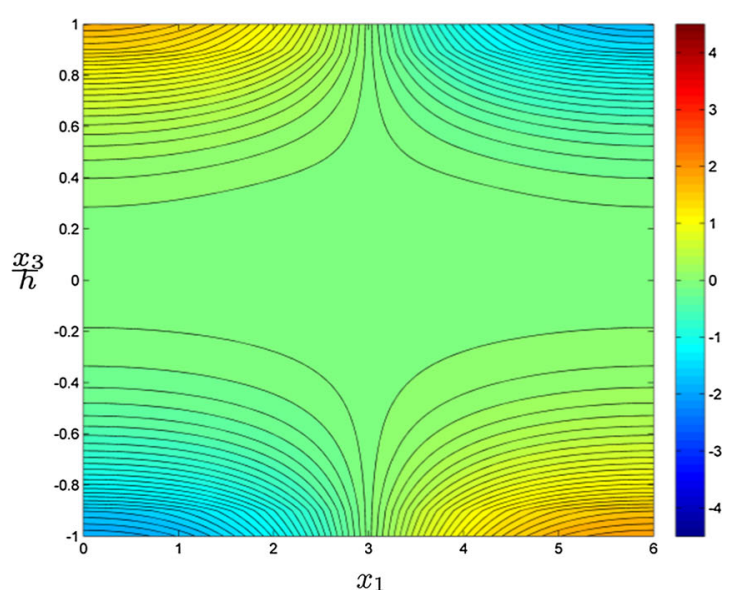

(b)

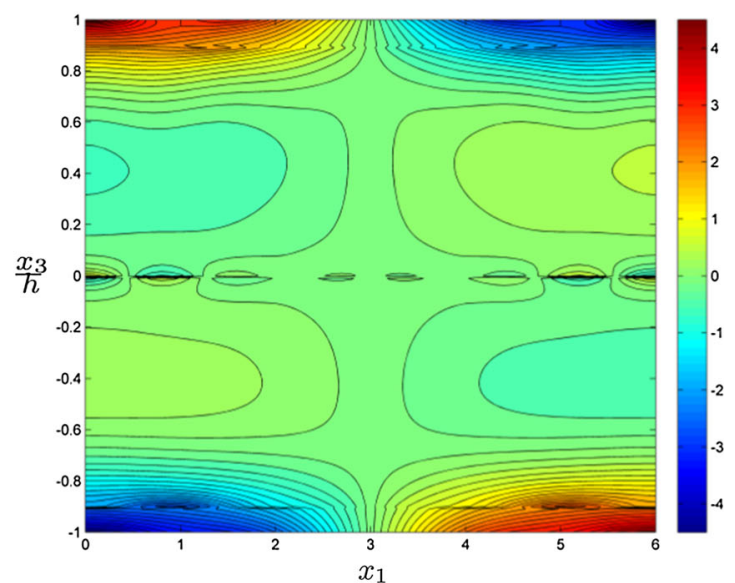

(d)

Fig. 8 Through-thickness normalised in-plane shear stress $\bar{\sigma}_{12}$ under sinusoidal and 'small' patch loadings on a central vertical section $x_{2}=0.5 b$ : a 'base material' under sinusoidal loading; b 'base material' under patch loading; $\mathbf{c}$ 'low $G_{t}$ material' under sinusoidal loading; d 'low $G_{t}$ material' under patch loading

To facilitate direct comparison of stresses and displacements, for the 'base material' and 'low $G_{t}$ material', normalisation is as follows: $\bar{u}_{i}=\frac{G_{p} u_{i}}{q_{0}^{\text {sinusoidal }} h}$, where $G_{p}$ is shear modulus in the plane of isotropy. Stresses are normalised as $\bar{\sigma}_{i j}=\frac{\sigma_{i j}}{q_{0}^{\text {sinusoidal }}}$. To avoid repetition, sections in the $x_{1}$-direction only are given here as due to the isotropy in the $x_{1}-x_{2}$ plane, plots in the $x_{2}$-direction would be identical.

Plots of normalised in-plane normal stress $\bar{\sigma}_{11}$ are shown in Fig. 7 on a vertical cross section taken at $x_{2}=0.5 b$. It can be seen that decreasing the transverse shear modulus of the core leads to an increase in the in-plane normal stresses within the face sheets. This is the case under both sinusoidal and patch loadings.

Figure 8 shows the distribution of normalised in-plane shear stress $\bar{\sigma}_{12}$ on a vertical section located at $x_{2}=0$ for panels under sinusoidal and patch loadings. A similar trend is observed in the plots of in-plane normal stress. Once again due to the low transverse shear modulus, the core offers very little shear resistance and the core and face sheets no longer work together. The panel with low transverse shear modulus (Fig. 8c, d) has individual peaks of in-plane shear stress within the upper and lower halves of the core.

Figure 9 shows the distribution of normalised out-of-plane normal stress $\bar{\sigma}_{33}$ on a vertical section located at $x_{2}=0.5 b$ for panels under sinusoidal and patch loadings. In the panels with lower transverse shear modulus, it can be seen that the stresses caused by the transverse loading penetrate further into the panel. For the panel with the highest transverse shear modulus, the stresses are higher (than the panels with lower transverse modulus) in the upper half of the panel but lower in the bottom half. Similarly, in the lower half of the panel the stresses 


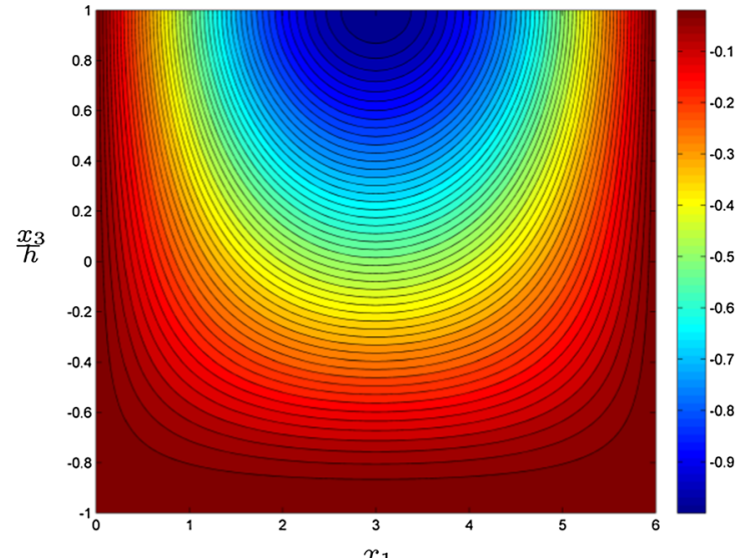

(a)

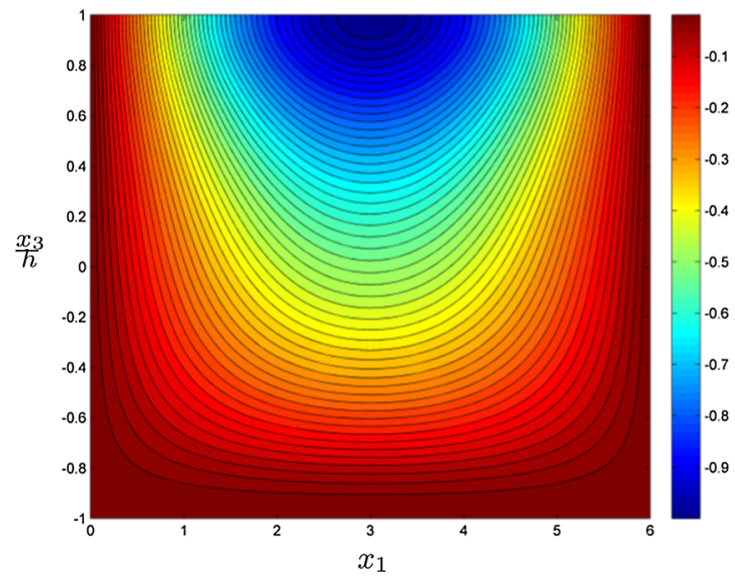

(c)

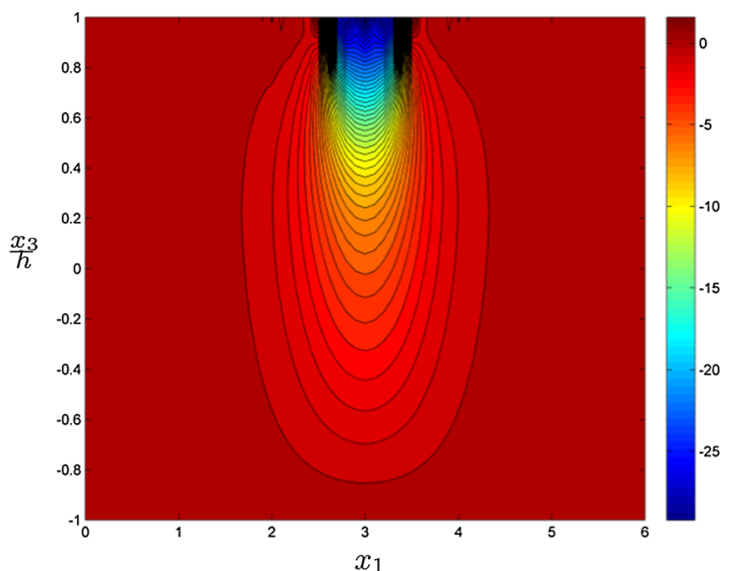

(b)

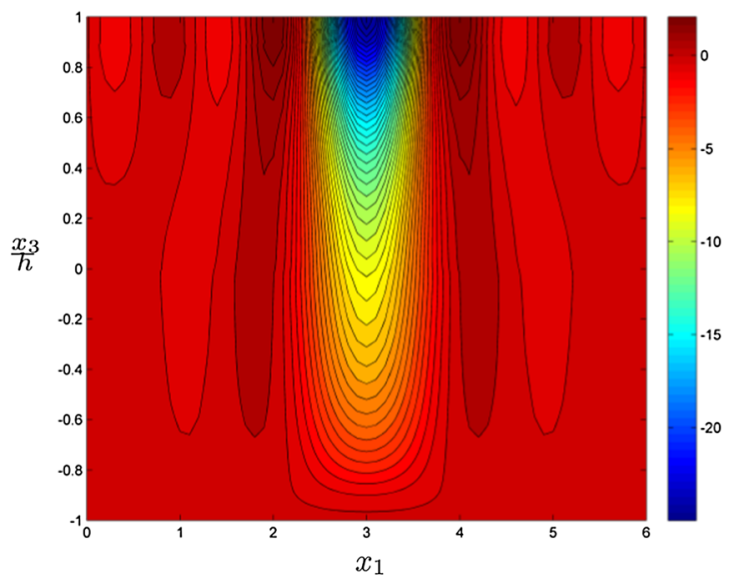

(d)

Fig. 9 Through-thickness variation of normal stress $\bar{\sigma}_{33}$ under sinusoidal and 'small' patch loadings on a central vertical section $x_{2}=0.5 b$ : a 'base material' under sinusoidal loading; $\mathbf{b}$ 'base material' under patch loading; c 'low $G_{t}$ material' under sinusoidal loading; d 'low $G_{t}$ material' under patch loading

for the panel with lowest transverse shear modulus are higher (than the panels with higher transverse shear modulus).

Figure 10 shows the distribution of normalised transverse shear stress $\bar{\sigma}_{13}$ through the panel with a section taken through the panel centre. In the panel with base transverse shear modulus (Fig. 10a), as was previously seen for the panels with isotropic core [17] the core can once more be seen to be carrying the majority of the transverse shear stresses, with variation taking an almost parabolic form through the thickness. The stress is again anti-symmetric around the panel centre with maximum and minimum stresses being equal in magnitude but opposite in sign located at $x_{1}=a$ and $x_{1}=0$, respectively. Equally, under patch loading (Fig. 10b), it is clearly seen that the maximum transverse shear stress is once again located (in the $x_{1}$-direction) below the boundary of where patch loading is applied. It can also be seen that the transverse shear stress is concentrated in the upper section of the core leaving the majority of the lower core free of transverse shear stress. For the panel with low transverse shear modulus, the effects of shear deformation can be observed. The transverse shear stress no longer varies from a minimum at the outer edges of the face sheets to a maximum at the panel centre. Under both sinusoidal (Fig. 10c) and patch (Fig. 10d) loadings, the face sheets can be seen to be carrying a greater proportion of the shear stresses, whilst shear stresses at the centre of the core are reduced. Under patch loading, it should also be noted that the lower half of the core now also shows localised effects under the edges of the patch loading. Since the main purpose of the core is to carry the shear stresses, reducing the shear carrying capacity of the core and transferring it to the face sheets would not be advantageous.

Figure 11 shows the distribution of von Mises stress on the panel. As the transverse shear modulus is reduced, the increase in in-plane normal stresses becomes particularly apparent. The increased bending associated with 


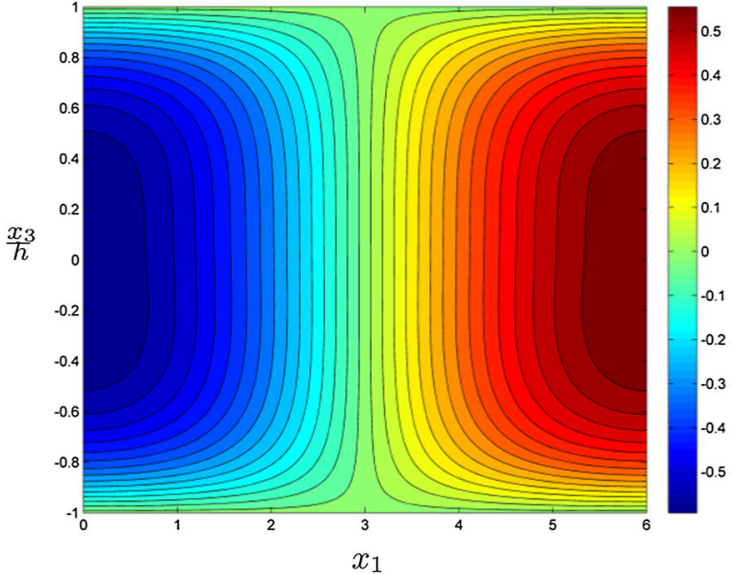

(a)

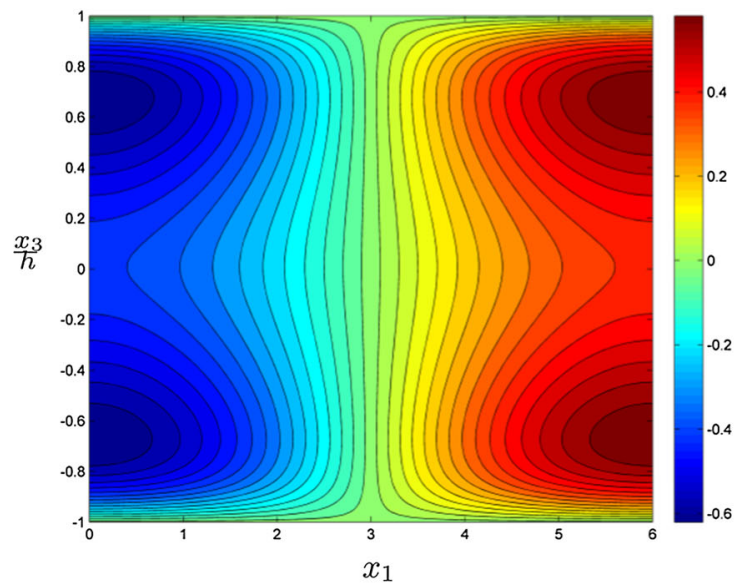

(c)

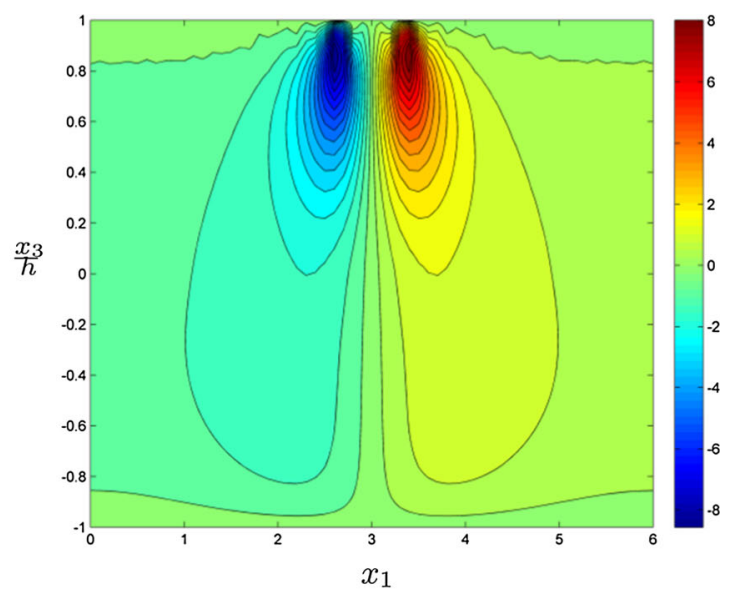

(b)

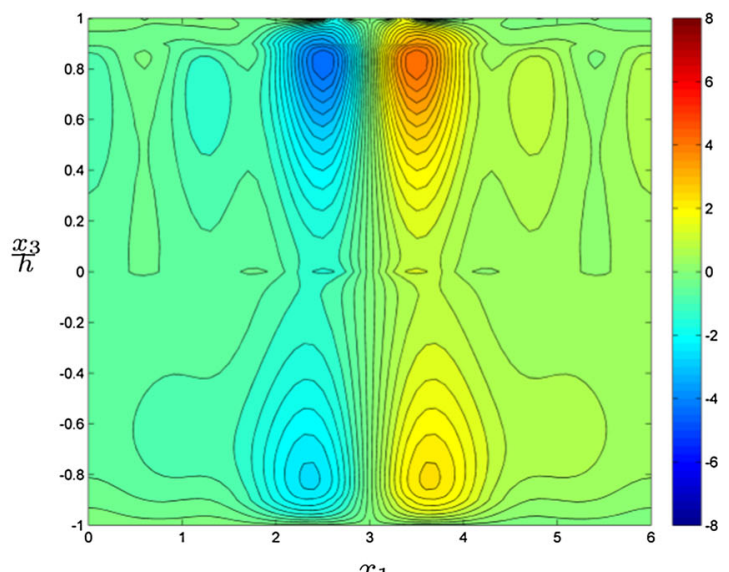

(d)

Fig. 10 Through-thickness variation of normalised transverse shear stress $\bar{\sigma}_{13}$ under sinusoidal and patch loadings on a central vertical section $x_{2}=0.5 b$ : a 'base material' under sinusoidal loading; $\mathbf{b}$ 'base material' under patch loading; c 'low $G_{t}$ material' under sinusoidal loading; $\mathbf{d}$ 'low $G_{t}$ material' under patch loading

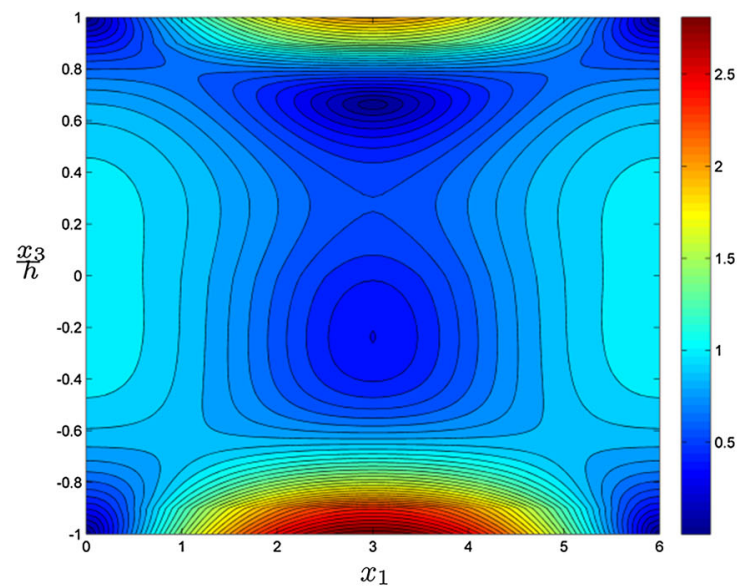

(a)

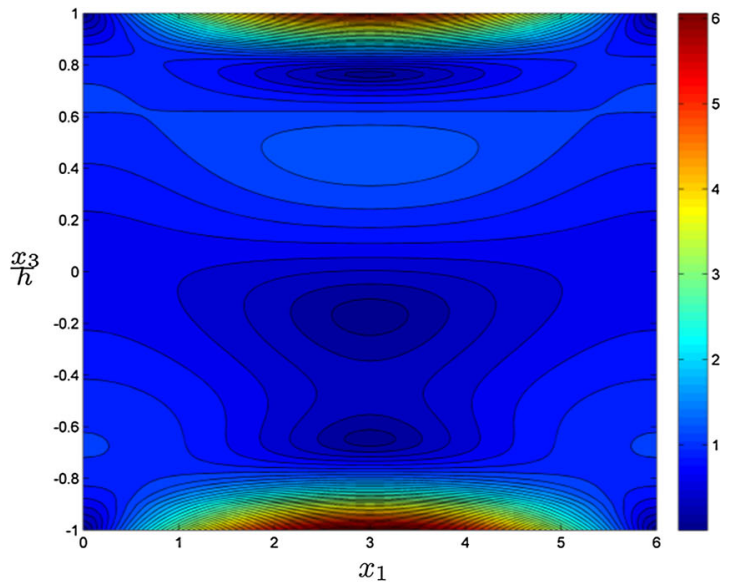

(b)

Fig. 11 Through-thickness variation of von Mises stress $\bar{\sigma}_{v o n}$ on a central vertical section $x_{2}=b / 2$ : a 'base material' under sinusoidal loading; b 'low $G_{t}$ material' under sinusoidal loading 


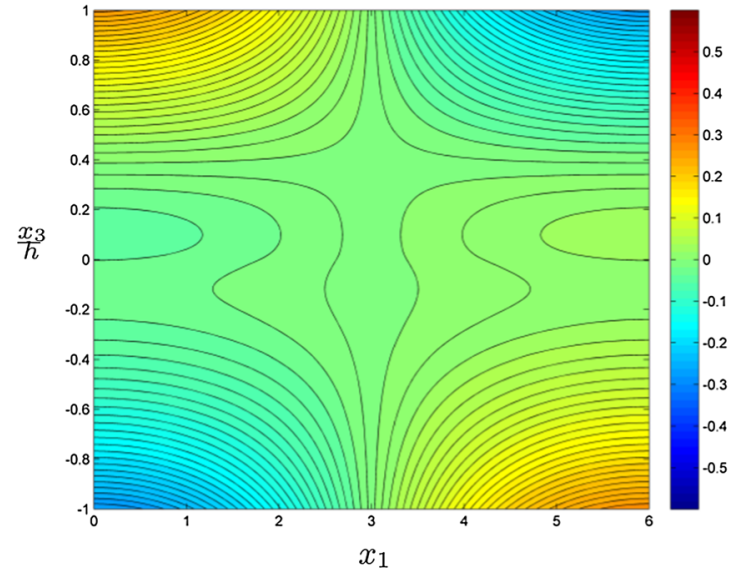

(a)

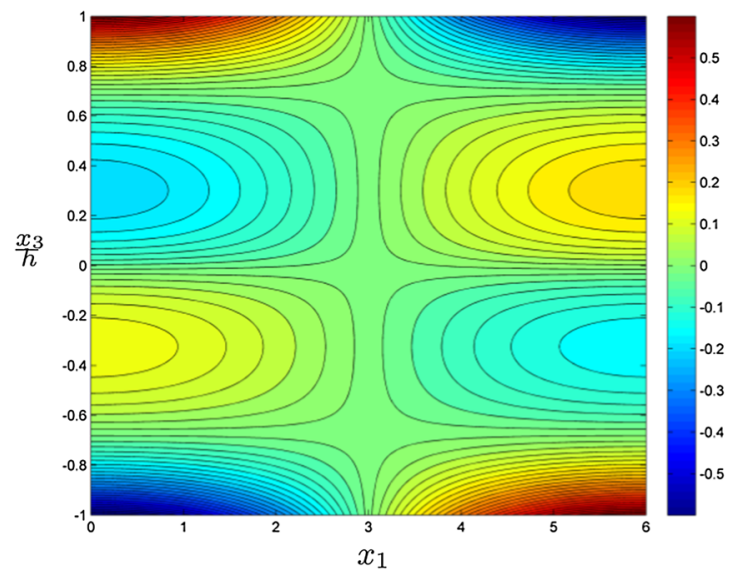

(c)

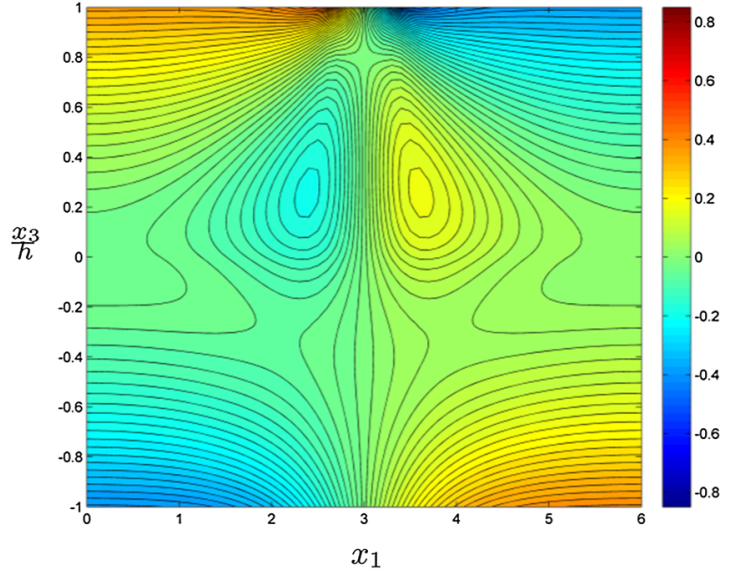

(b)

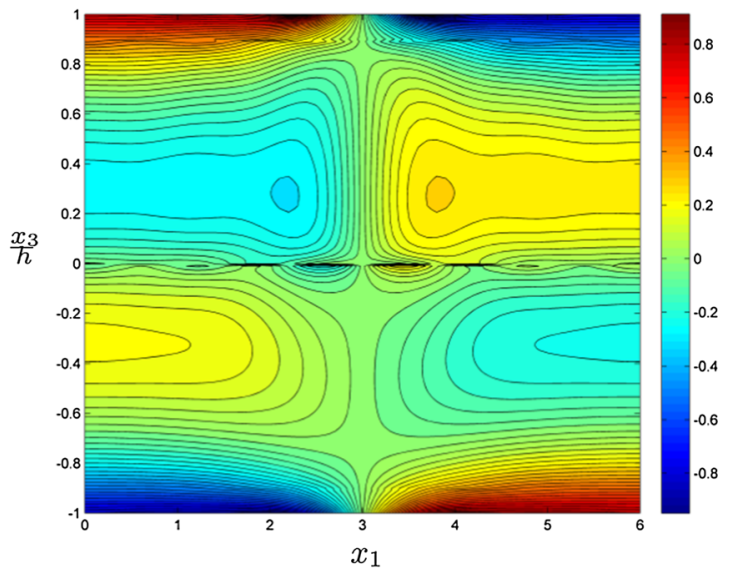

(d)

Fig. 12 Through-thickness variation normalised in-plane displacement $\bar{u}_{1}$ under sinusoidal and patch loadings on a central vertical section $x_{2}=0.5 b$ : a 'base material' under sinusoidal loading; b 'base material' under patch loading; c 'low $G_{t}$ material' under sinusoidal loading; d 'low $G_{t}$ material' under patch loading

low transverse shear modulus is a particular danger as it may give rise to core buckling, face sheet wrinkling or failure of the core in shear.

Figure 12 shows the distribution of normalised in-plane displacement $\bar{u}_{1}$ on a central vertical section $x_{2}=0.5 b$ under sinusoidal and patch loadings. For the panels with low transverse shear modulus (Fig. 12c, d), the core can be seen to deform more easily than the panels with base transverse shear modulus.

Study of the normalised transverse displacement $\bar{u}_{3}$ (Fig. 13) shows a large increase in displacement as the transverse shear modulus is decreased due to the increased panel bending. This is observed for both sinusoidal (Fig. 13a, c) and patch loadings (Fig. 13b, d).

\subsection{Effect of anisotropy in thick and thin panels}

In order to study the effect of shear deformation in greater detail, a comparison is made between a panel with transversely isotropic 'low $G_{t}$ material' and a panel whose coefficients have been modified to give an equivalent isotropic panel, with properties listed in Table 3 under 'Equivalent Isotropic material'.

Plots of normalised transverse shear stress on a line through the panel thickness at $x_{1}=0, x_{2}=0.5 b$ are shown in Fig. 14. Four different panel thicknesses are considered: very thick panels with $a / h_{0}=b / h_{0}=1.5$; thick panels with $a / h_{0}=b / h_{0}=2$; moderately thin panels with $a / h_{0}=b / h_{0}=6$ and thin panels with $a / h_{0}=b / h_{0}=10$. 


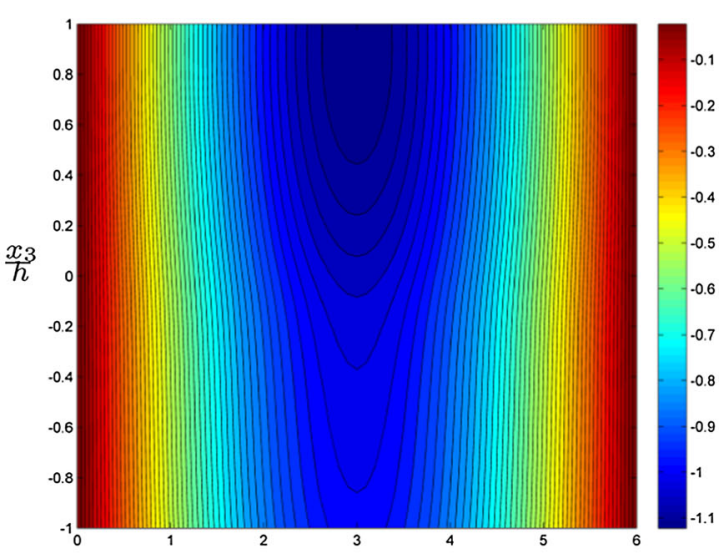

$x_{1}$

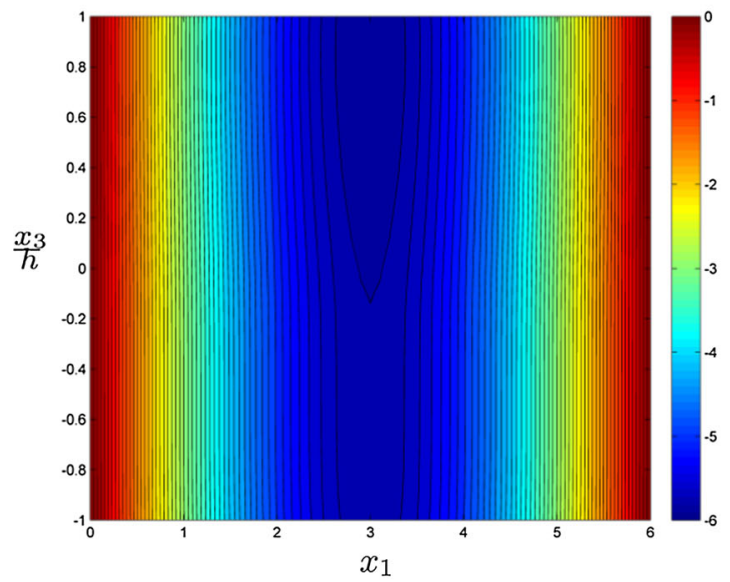

(c)

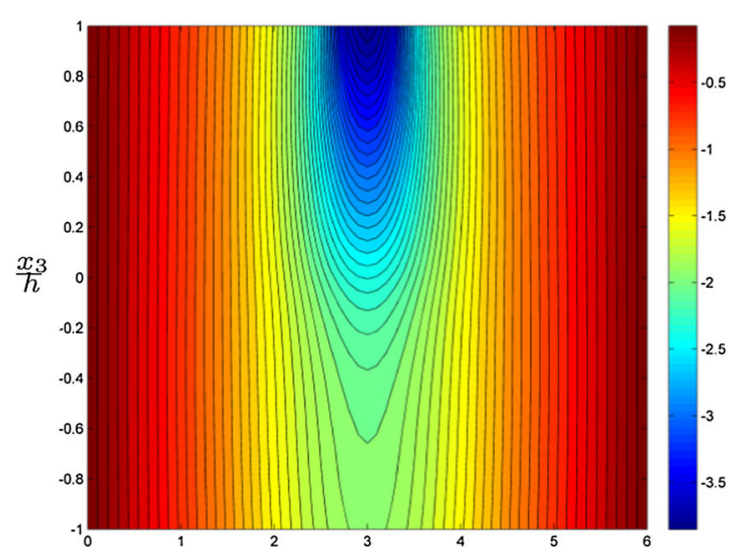

(b)

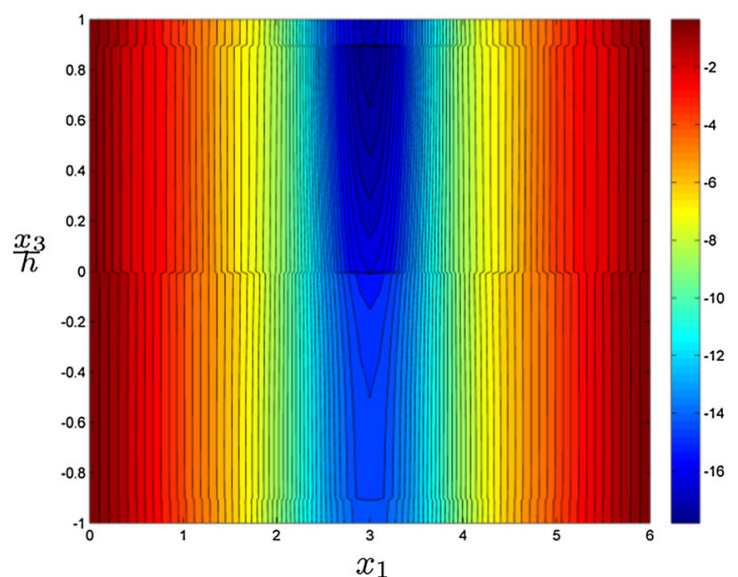

(d)

Fig. 13 Through-thickness variation of normalised transverse displacement $\bar{u}_{3}$ under sinusoidal and patch loadings on a central vertical section $x_{2}=0.5 b$ : a 'base material' under sinusoidal loading; b 'base material' under patch loading; c 'low $G_{t}$ material' under sinusoidal loading; d 'low $G_{t}$ material' under patch loading

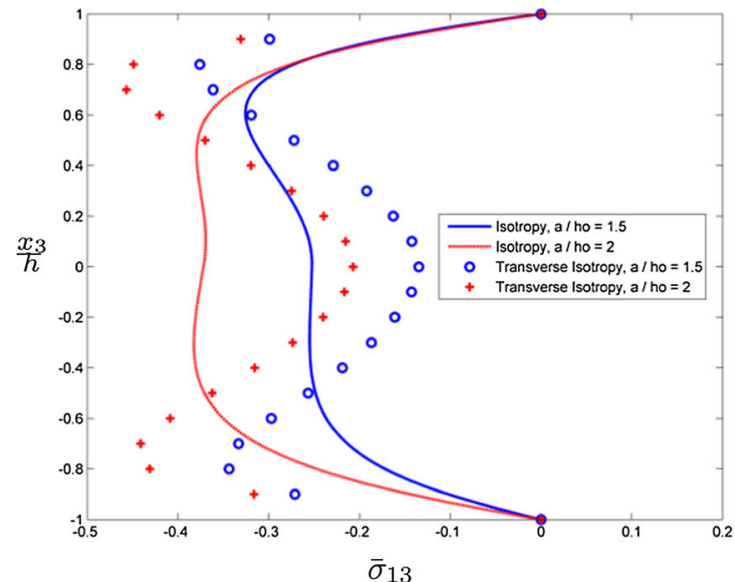

(a)

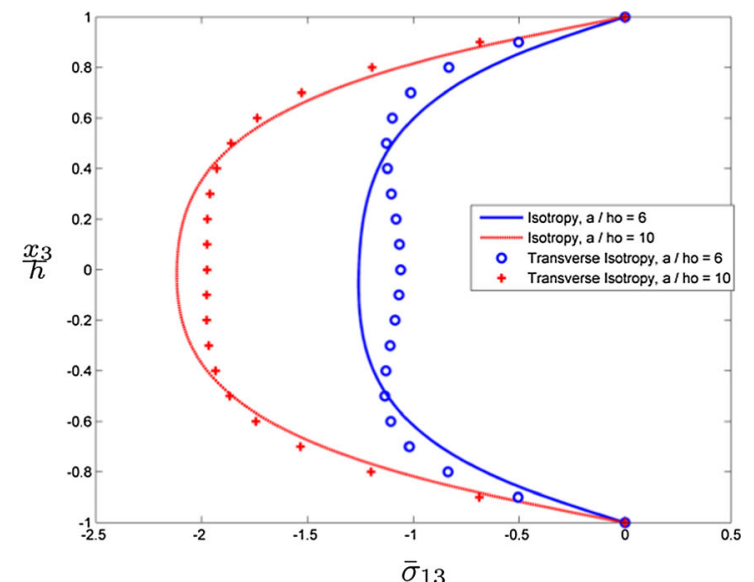

(b)

Fig. 14 Effect of isotropy and panel thickness on through-thickness variation of normalised transverse shear stress $\bar{\sigma}_{13}$ at $x_{1}=$ $0, x_{2}=0.5 b$ 
When considering the thick and very thick panels (Fig. 14a), it can be seen that for the transversely isotropic panel, the through-thickness variation of transverse shear stress is non-parabolic and the maximum occurs close to the upper/lower face sheet, pointing to a high degree of shear deformation. For the isotropic panel, it can be seen that for the thick and very thick panels, the shear response is much more parabolic in shape, but shear deformation is just starting to become apparent.

Conversely, when considering the moderately thin and thin panels (Fig. 14b), it can be seen that for the transversely isotropic panel, shear deformation is still evident even when $a / h_{0}=b / h_{0}=10$. However, for the isotropic panel, the response is parabolic for both the moderately thin and thin panels. It has been shown that shear deformation is apparent in transversely isotropic panels at much smaller thickness to span ratio than an equivalent isotropic panel and as such when designing panels with transversely isotropic core material, shear deformation effects should not be ignored even for moderately thin and thin panels. This is especially true for panels with lower transverse shear modulus.

\section{Concluding remarks}

In this paper, a three-dimensional elasticity solution was developed for simply supported sandwich panels with transversely isotropic functionally graded core subjected to transverse loading. The Young's and shear moduli of the core material were assumed to vary exponentially through the thickness, whilst the Poisson's ratios were assumed to remain constant. The solution was derived using displacement functions for inhomogeneous transversely isotropic media proposed in [18] and was validated through comparison with results for an isotropic functionally graded plate [17] and a homogenous transversely isotropic plate [32].

The solution allowed the first full 3D analysis of sandwich panels with transversely isotropic graded core to be carried out. Key findings of the study were as follows:

- In panels with low transverse shear modulus, a greater effect of shear deformation was observed both under distributed and localised loadings;

- A comparative study of panels with transversely isotropic and isotropic graded cores demonstrated that when considering the panel with transversely isotropic core, the effects of shear deformation are apparent in thinner panels with much smaller thickness to span ratio than in an equivalent isotropic panel;

- In panels with transversely isotropic core highly non-parabolic variation of transverse shear stress was observed;

- Increasing the in-plane Young's modulus was shown to give the panel greater resistance to bending and a reduction in displacement throughout for both thin and thick panels;

- Under localised loading, it was seen that high displacement gradients can occur in areas close to the load application. The functionally graded core was seen to provide additional resistance under areas of localised loading, reducing the load penetration through the panel thickness. This is important practically, as indentation of the core would reduce the cross-sectional area of the panel and therefore decrease the bending stiffness of the panel as a whole. In addition, the graded core gave a reduction in the magnitude of shear and normal stresses near to the load application. This provides the panel with greater resistance to failure of the core material by yielding or shear.

- Local bending of the upper face sheet under localised transverse loading was also shown to be eliminated through introduction of the graded core as the effects of the local load can be effectively distributed across the interface into the panel core.

Acknowledgements Financial support of this research by the EPSRC DTA is gratefully acknowledged.

Open Access This article is distributed under the terms of the Creative Commons Attribution 4.0 International License (http:// creativecommons.org/licenses/by/4.0/), which permits unrestricted use, distribution, and reproduction in any medium, provided you give appropriate credit to the original author(s) and the source, provide a link to the Creative Commons license, and indicate if changes were made. 


\section{Appendix}

Functions $U_{i j}^{(k)}$ and $P_{r t j}^{(k)}$ involved in Eqs. (17a-17i) are

$$
\begin{aligned}
& U_{1 j}^{(k)}\left(m, n, x_{3}\right)=-\frac{q_{m n} h}{c_{11}^{0} c_{33}^{0}-c_{13}^{02}} \frac{\pi m h}{a} \exp \left(\frac{-\alpha^{(k)} x_{3}}{h}\right)\left[c_{13}^{0} k_{\Psi}^{2} h^{2} f_{j}\left(x_{3}\right)+c_{33}^{0} h^{2} \frac{\mathrm{d}^{2}}{\mathrm{~d} x_{3}^{2}} f_{j}\left(x_{3}\right)\right] \\
& U_{2 j}^{(k)}\left(m, n, x_{3}\right)=-\frac{q_{m n} h}{c_{11}^{0} c_{33}^{0}-c_{13}^{02}} \frac{\pi n h}{b} \exp \left(\frac{-\alpha^{(k)} x_{3}}{h}\right)\left[c_{13}^{0} k_{\Psi}^{2} h^{2} f_{j}\left(x_{3}\right)+c_{33}^{0} h^{2} \frac{\mathrm{d}^{2}}{\mathrm{~d} x_{3}^{2}} f_{j}\left(x_{3}\right)\right] \\
& U_{3 j}^{(k)}\left(m, n, x_{3}\right)=-\frac{q_{m n} h}{c_{11}^{0} c_{33}^{0}-c_{13}^{02}} \exp \left(\frac{-\alpha^{(k)} x_{3}}{h}\right)\left[c_{33}^{0}\left(-h^{3} \frac{\mathrm{d}^{3}}{\mathrm{~d} x_{3}^{3}} f_{j}\left(x_{3}\right)-\alpha^{(k)} \frac{\mathrm{d}^{2}}{\mathrm{~d} x_{3}^{2}} f_{j}\left(x_{3}\right)\right)\right. \\
& \left.+k_{\Psi}^{2} h^{2}\left(h\left(\frac{c_{11}^{0} c_{33}^{0}}{c_{44}^{0}}-c_{13}^{0}-\frac{c_{13}^{02}}{c_{44}^{0}}\right) \frac{\mathrm{d}}{\mathrm{d} x_{3}} f_{j}\left(x_{3}\right)+\alpha^{(k)} c_{13}^{0} f_{j}\left(x_{3}\right)\right)\right] \\
& j=1, \ldots, 4 ; \\
& U_{1 j}^{(k)}\left(m, n, x_{3}\right)=-\frac{q_{m n} h}{c_{44}^{0}} \frac{\pi n h}{b} f_{j}\left(x_{3}\right) \\
& U_{2 j}^{(k)}\left(m, n, x_{3}\right)=\frac{q_{m n} h}{c_{44}^{0}} \frac{\pi m h}{a} f_{j}\left(x_{3}\right) \\
& U_{3 j}^{(k)}\left(m, n, x_{3}\right)=0 \\
& j=5,6 \text {; } \\
& P_{33 j}^{(k)}\left(m, n, x_{3}\right)=q_{m n} k_{\Psi}^{4} h^{4} f_{j}\left(x_{3}\right) \\
& P_{13 j}^{(k)}\left(m, n, x_{3}\right)=q_{m n} k_{\Psi}^{2} h^{3}\left(\frac{\pi m h}{a}\right) \frac{\mathrm{d}}{\mathrm{d} x_{3}} f_{j}\left(x_{3}\right) \\
& P_{23 j}^{(k)}\left(m, n, x_{3}\right)=q_{m n} k_{\Psi}^{2} h^{3}\left(\frac{\pi n h}{b}\right) \frac{\mathrm{d}}{\mathrm{d} x_{3}} f_{j}\left(x_{3}\right) \\
& P_{11 j}^{(k)}\left(m, n, x_{3}\right)=q_{m n}\left[\frac{c_{11}^{0} c_{33}^{0}-c_{12}^{0} c_{13}^{0}}{c_{11}^{0} c_{33}^{0}-c_{13}^{02}} k_{\Psi}^{2} h^{2}\left(\frac{\pi n h}{b}\right)^{2} f_{j}\left(x_{3}\right)\right. \\
& \left.-\frac{c_{12}^{0} c_{33}^{0}-c_{13}^{02}}{c_{11}^{0} c_{33}^{0}-c_{13}^{02}} h^{2}\left(\frac{\pi n h}{b}\right)^{2} \frac{\mathrm{d}^{2}}{\mathrm{~d} x_{3}^{2}} f_{j}\left(x_{3}\right)-h^{2}\left(\frac{\pi m h}{a}\right)^{2} \frac{\mathrm{d}^{2}}{\mathrm{~d} x_{3}^{2}} f_{j}\left(x_{3}\right)\right] \\
& P_{22 j}^{(k)}\left(m, n, x_{3}\right)=q_{m n}\left[\frac{c_{11}^{0} c_{13}^{0}-c_{12}^{0} c_{13}^{0}}{c_{11}^{0} c_{33}^{0}-c_{13}^{02}} k_{\Psi}^{2} h^{2}\left(\frac{\pi m h}{a}\right)^{2} f_{j}\left(x_{3}\right)\right. \\
& \left.-\frac{c_{12}^{0} c_{33}^{0}-c_{13}^{02}}{c_{11}^{0} c_{33}^{0}-c_{13}^{02}} h^{2}\left(\frac{\pi m h}{a}\right)^{2} \frac{\mathrm{d}^{2}}{\mathrm{~d} x_{3}^{2}} f_{j}\left(x_{3}\right)-h^{2}\left(\frac{\pi n h}{b}\right)^{2} \frac{\mathrm{d}^{2}}{\mathrm{~d} x_{3}^{2}} f_{j}\left(x_{3}\right)\right] \\
& P_{12 j}^{(k)}\left(m, n, x_{3}\right)=q_{m n} \frac{c_{11}^{0}-c_{12}^{0}}{c_{11}^{0} c_{33}^{0}-c_{13}^{02}}\left(\frac{\pi m h}{a}\right)\left(\frac{\pi n h}{b}\right) h^{2}\left[c_{13}^{0} k_{\Psi}^{2} f_{j}\left(x_{3}\right)+c_{33}^{0} \frac{\mathrm{d}^{2}}{\mathrm{~d} x_{3}^{2}} f_{j}\left(x_{3}\right)\right] \\
& j=1, \ldots, 4 \text {; } \\
& P_{33 j}^{(k)}\left(m, n, x_{3}\right)=0 \\
& P_{13 j}^{(k)}\left(m, n, x_{3}\right)=-q_{m n}\left(\frac{\pi n h}{b}\right) \exp \left(\frac{\alpha^{(k)} x_{3}}{h}\right) \frac{\mathrm{d}}{\mathrm{d} x_{3}} f_{j}\left(x_{3}\right) \\
& P_{23 j}^{(k)}\left(x_{3}\right)=q_{m n}\left(\frac{\pi m h}{a}\right) \exp \left(\frac{\alpha^{(k)} x_{3}}{h}\right) \frac{\mathrm{d}}{\mathrm{d} x_{3}} f_{j}\left(x_{3}\right)
\end{aligned}
$$




$$
\begin{aligned}
P_{11 j}^{(k)}\left(x_{3}\right)= & \frac{q_{m n}}{c_{44}^{0}} \frac{1}{c_{11}^{0} c_{33}^{0}-c_{13}^{02}}\left(\frac{\pi n h}{b}\right)\left(\frac{\pi m h}{a}\right) \exp \left(\frac{\alpha^{(k)} x_{3}}{h}\right) \\
& \times\left(c_{12}^{0} c_{13}^{02}+c_{11}^{02} c_{33}^{0}-c_{11}^{0} c_{13}^{02}-c_{12}^{0} c_{11}^{0} c_{33}^{0}\right) f_{j}\left(x_{3}\right) \\
P_{22 j}^{(k)}\left(x_{3}\right)= & \frac{q_{m n}}{c_{44}^{0}} \frac{1}{c_{11}^{0} c_{33}^{0}-c_{13}^{02}}\left(\frac{\pi n h}{b}\right)\left(\frac{\pi m h}{a}\right) \exp \left(\frac{\alpha^{(k)} x_{3}}{h}\right) \\
& \times\left(-c_{12}^{0} c_{13}^{02}-c_{11}^{02} c_{33}^{0}+c_{11}^{0} c_{13}^{02}+c_{12}^{0} c_{11}^{0} c_{33}^{0}\right) f_{j}\left(x_{3}\right) \\
P_{12 j}^{(k)}\left(x_{3}\right)= & \frac{q_{m n}}{2} \frac{c_{11}^{0}-c_{12}^{0}}{c_{44}^{0}}\left[\left(\frac{\pi m h}{a}\right)^{2}-\left(\frac{\pi n h}{b}\right)^{2}\right] \exp \left(\frac{\alpha^{(k)} x_{3}}{h}\right) f_{j}\left(x_{3}\right) \\
j= & 5,6 .
\end{aligned}
$$

In the expressions above, functions $f_{j}\left(x_{3}\right), \quad j=1, \ldots, 4$ are as follows.

If discriminant of characteristic equation is negative,

$$
\begin{aligned}
& f_{1}\left(x_{3}\right)=\exp \left(\frac{\alpha x_{3}}{2 h}\right) \cosh \frac{\lambda x_{3}}{h} \cosh \frac{\mu x_{3}}{h}, \\
& f_{2}\left(x_{3}\right)=\exp \left(\frac{\alpha x_{3}}{2 h}\right) \sinh \frac{\lambda x_{3}}{h} \cosh \frac{\mu x_{3}}{h} \\
& f_{1}\left(x_{3}\right)=\exp \left(\frac{\alpha x_{3}}{2 h}\right) \cosh \frac{\lambda x_{3}}{h} \sinh \frac{\mu x_{3}}{h} \\
& f_{2}\left(x_{3}\right)=\exp \left(\frac{\alpha x_{3}}{2 h}\right) \sinh \frac{\lambda x_{3}}{h} \sinh \frac{\mu x_{3}}{h}
\end{aligned}
$$

If discriminant of characteristic equation is positive with four real roots,

$$
\begin{array}{ll}
f_{1}\left(x_{3}\right)=\exp \left(\frac{\alpha x_{3}}{2 h}\right) \cosh \frac{\lambda x_{3}}{h}, & f_{1}\left(x_{3}\right)=\exp \left(\frac{\alpha x_{3}}{2 h}\right) \sinh \frac{\lambda x_{3}}{h}, \\
f_{1}\left(x_{3}\right)=\exp \left(\frac{\alpha x_{3}}{2 h}\right) \cosh \frac{\mu x_{3}}{h}, & f_{1}\left(x_{3}\right)=\exp \left(\frac{\alpha x_{3}}{2 h}\right) \sinh \frac{\mu x_{3}}{h}
\end{array}
$$

If the discriminant of the characteristic equation is positive with two real roots and two complex roots, then

$$
\begin{array}{ll}
f_{1}\left(x_{3}\right)=\exp \left(\frac{\alpha x_{3}}{2 h}\right) \cosh \frac{\lambda x_{3}}{h}, & f_{2}\left(x_{3}\right)=\exp \left(\frac{\alpha x_{3}}{2 h}\right) \sinh \frac{\lambda x_{3}}{h}, \\
f_{3}\left(x_{3}\right)=\exp \left(\frac{\alpha x_{3}}{2 h}\right) \cosh \frac{\mu x_{3}}{h}, & f_{4}\left(x_{3}\right)=\exp \left(\frac{\alpha x_{3}}{2 h}\right) \sinh \frac{\mu x_{3}}{h}
\end{array}
$$

If the discriminant of the characteristic equation is positive with four complex roots, then

$$
\begin{array}{ll}
f_{1}\left(x_{3}\right)=\exp \left(\frac{\alpha x_{3}}{2 h}\right) \cosh \frac{\lambda x_{3}}{h}, & f_{2}\left(x_{3}\right)=\exp \left(\frac{\alpha x_{3}}{2 h}\right) \sinh \frac{\lambda x_{3}}{h}, \\
f_{3}\left(x_{3}\right)=\exp \left(\frac{\alpha x_{3}}{2 h}\right) \cosh \frac{\mu x_{3}}{h}, & f_{4}\left(x_{3}\right)=\exp \left(\frac{\alpha x_{3}}{2 h}\right) \sinh \frac{\mu x_{3}}{h} ;
\end{array}
$$

Functions $f_{5}\left(x_{3}\right)$ and $f_{6}\left(x_{3}\right)$ are

$$
f_{5}\left(x_{3}\right)=\exp \left(\frac{\alpha x_{3}}{2 h}\right) \cosh \frac{\beta x_{3}}{h}, \quad f_{6}\left(x_{3}\right)=\exp \left(\frac{-\alpha x_{3}}{2 h}\right) \sinh \frac{\beta x_{3}}{h}
$$




\section{References}

1. Abrate, S.: Impact on Composite Structures. Cambridge University Press, New York (1998)

2. Akavci, S.S.: Mechanical behaviour of functionally graded plates on elastic foundation. Compos. B Eng. 96, 136-152 (2016)

3. Alibeigloo, A.: Three-dimensional thermo-elasticity solution of sandwich cylindrical panel with functionally graded core. Compos. Struct. 107, 458-468 (2014)

4. Alibeigloo, A., Liew, K.M.: Free vibration analysis of sandwich cylindrical panel with functionally graded core using threedimensional theory of elasticity. Compos. Struct. 113, 23-30 (2014)

5. Anderson, T.A.: A 3-D elasticity solution for a sandwich composite with functionally graded core subjected to transverse loading by a rigid sphere. Compos. Struct. 60, 265-74 (2003)

6. Apetre, N.A., Sankar, B.V., Ambur, D.R.: Analytical modeling of sandwich beams with functionally graded core. J. Sandw. Struct. Mater. 10, 53-74 (2008)

7. Birman, V., Byrd, L.W.: Modelling and analysis of functionally graded materials and structures. Appl. Mech. Rev. 60, 195-216 (2007)

8. Birman, V., Costa, H.: Wrinkling of functionally graded sandwich structures subject to biaxial and in-plane shear loads. ASME J. Appl. Mech. 84, 121006-1-121006-10 (2017)

9. Birman, V., Kardomateas, G.A.: Review of current trends in research and applications of sandwich structures. Compos. Struct. 142, 221-240 (2018)

10. Birman, V., Vo, N.: Winkling in sandwich structures with functionally graded core. ASME J. Appl. Mech. 84, 021002-1021002-8 (2017)

11. Brischetto, S.: Exponential matrix method for the solutions of exact 3D equilibrium equations for free vibrations of functionally graded plates and shells. J. Sandw. Struct. Mater. 21, 77-114 (2019)

12. Daynes, S., Feih, S., Lu, W.F., Wei, J.: Optimisation of functionally graded lattice structures using isostatic lines. Mater. Des. 127, 2152-223 (2017)

13. Ding, H.J., Chen, W., Zhang, L.: Elasticity of Transversely Isotropic Materials, 1st edn. Springer, Dordrecht (2006)

14. Etemadi, E., Afaghi Khatibi, A., Takaffoli, M.: 3D finite element simulation of sandwich panels with a functionally graded core subjected to low velocity impact. Compos. Struct. 89, 28-34 (2009)

15. Frostig, Y., Birman, V., Kardomateas, G.A.: Non-linear wrinkling of a sandwich panel with functionally graded coreextended high-order approach. Int. J. Solids Struct. 148-149, 122-139 (2018)

16. Kashtalyan, M.: Three-dimensional elasticity solution for bending of functionally graded rectangular plates. Eur. J. Mech. A/Solids 23, 853-64 (2004)

17. Kashtalyan, M., Menshykova, M.: Three-dimensional elasticity solution for sandwich panels with a functionally graded core. Compos. Struct. 87, 36-43 (2009)

18. Kashtalyan, M., Rushchitsky, J.J.: Revisiting displacement functions in three-dimensional elasticity theory of inhomogeneous media. Int. J. Solids Struct. 46, 3463-3470 (2009)

19. Kelly, M., Arora, H., Worley, A., Kaye, M., Del Linz, P., Hopper, P.A., Dear, J.P.: Sandwich panel cores for blast applications: materials and graded density. Exper. Mech. 56, 523-544 (2016)

20. Kirugulige, M.S., Kitey, R., Tippur, H.V.: Dynamic fracture behaviour of model sandwich structures with functionally graded core: a feasibility study. Compos. Sci. Technol. 65, 1052-1068 (2005)

21. Liu, X.R., Tian, X.G., Lu, T.J., Liang, B.: Sandwich plates with functionally graded metallic foam cores subjected to air blast loading. Int. J. Mech. Sci. 84, 61-72 (2014)

22. Plevako, V.P.: On the theory of elasticity of inhomogeneous media. J. Appl. Math. Mech. 35, 806-813 (1971)

23. Rahmani, O., Khalili, S.M.R., Malekzadeh, K., Hadavinia, H.: Free vibration analysis of sandwich structures with a flexible functionally graded syntactic core. Compos. Struct. 91, 229-235 (2009)

24. Sburlati, R.: An axisymmetric elastic analysis for circular sandwich panels with functionally graded cores. Compos. B Eng. 43, 1039-1044 (2012)

25. Sburlati, R., Atashipour, S.R., Atashipour, S.A.: Exact elastic analysis of a doubly coated thick circular plate using functionally graded interlayers. Arch. Appl. Mech. 85, 1779-1792 (2015)

26. Tokovyy, Y.V., Ma, C.C.: Three-dimensional elastic analysis of transversely isotropic composites. J. Mech. 33, 821-830 (2017)

27. Tokovyy, Y.V.: Direct integration of three-dimensional thermoelasticity equations for a transversely isotropic layer. J. Therm. Stress. 42, 49-64 (2019)

28. Vescovini, R., D’Ottavio, M., Dozio, L., Polit, O.: Buckling and wrinkling of anisotropic sandwich plates. Int. J. Eng. Sci. 130, 136-156 (2018)

29. Wang, E., Gardner, N., Shukla, A.: The blast resistance of sandwich composites with stepwise graded cores. Int. J. Solids Struct. 46, 3492-3502 (2009)

30. Woodward, B., Kashtalyan, M.: Bending response of sandwich panels with graded core: 3D elasticity analysis. Mech. Adv. Mater. Struct. 178, 586-594 (2010)

31. Woodward, B., Kashtalyan, M.: 3D elasticity analysis of sandwich panels with graded core under distributed and concentrated loadings. Int. J. Mech. Sci. 53, 872-885 (2011a)

32. Woodward, B., Kashtalyan, M.: Three-dimensional elasticity solution for bending of transversely isotropic functionally graded plates. Eur. J. Mech. A/Solids 30, 705-718 (2011b)

33. Woodward, B., Kashtalyan, M.: Finite element modelling of sandwich panels with graded core under various boundary conditions. Aeronaut. J. 1186, 1285-1310 (2012)

34. Woodward, B., Kashtalyan, M.: A piecewise-exponential model for analysis of elastic deformation of sandwich panels with arbitrarily graded core. Int. J. Solids Struct. 75, 188 (2015)

35. Xiao, D., Mu, L., Zhao, G.: Influence of positive gradient metallic cellular core on energy dissipation of sandwich panels under indentation. Arch. Appl. Mech. 86, 1901-1911 (2016) 
36. Xu, G., Zhai, J., Zeng, T., Wang, Z., Cheng, S., Fang, D.: Response of composite sandwich beams with graded lattice core. Compos. Struct. 119, 666-676 (2015)

37. Zhou, J., Guan, Z.W., Cantwell, W.J.: The impact response of graded foam sandwich structures. Compos. Struct. 97, 370-377 (2013)

38. Zhu, H., Sankar, B.V.: Analysis of sandwich TPS panel with functionally graded foam core by Galerkin method. Compos. Struct. 77, 280-287 (2007)

Publisher's Note Springer Nature remains neutral with regard to jurisdictional claims in published maps and institutional affiliations. 